\title{
REVIEWS
}

\section{Taking phototherapeutics from concept to clinical launch}

\author{
Brianna M. Vickerman (1) ${ }^{1}$, Emilia M. Zywot $\mathbb{D}^{2}$, Teresa K. Tarrant $\mathbb{D}^{3 凶}$ \\ and David S. Lawrence (iD) $1,2,4 凶$
}

Abstract | More than four decades have passed since the first example of a light-activated (caged) compound was described. In the intervening years, a large number of light-responsive derivatives have been reported, several of which have found utility under a variety of in vitro conditions using cells and tissues. Light-triggered bioactivity furnishes spatial and temporal control, and offers the possibility of precision dosing and orthogonal communication with different biomolecules. These inherent attributes of light have been advocated as advantageous for the delivery and/or activation of drugs at diseased sites for a variety of indications. However, the tissue penetrance of light is profoundly wavelength-dependent. Only recently have phototherapeutics that are photoresponsive in the optical window of tissue $(600-900 \mathrm{~nm})$ been described. This Review highlights these recent discoveries, along with their limitations and clinical opportunities. In addition, we describe preliminary in vivo studies of prospective phototherapeutics, with an emphasis on the path that remains to be navigated in order to translate light-activated drugs into clinically useful therapeutics. Finally, the unique attributes of phototherapeutics is highlighted by discussing several potential disease applications.

'Department of Chemistry, University of North Carolina, Chapel Hill, NC, USA.

${ }^{2}$ Division of Chemical Biology and Medicinal Chemistry, University of North Carolina, Chapel Hill, NC, USA.

${ }^{3}$ Department of Medicine, Duke University Medical Center, Durham, NC, USA.

${ }^{4}$ Department of Pharmacology, University of North Carolina, Chapel Hill, NC, USA.

$\otimes_{e-m a i l:}$

teresa.tarrant@duke.edu; lawrencd@email.unc.edu

https://doi.org/10.1038

s41570-021-00326-w
In 1908, the German physician/scientist Paul Ehrlich introduced the term 'magic bullet' to describe the concept of designing chemicals that target invading microbes while sparing the human host ${ }^{1}$. The use of the word 'bullet' alludes to aiming at a target with a gun and arises from Ehrlich's refrain that "we have to learn how to aim chemically" 2 . The idea of using chemistry to aim more precisely at the diseased site has been expanded to include drugs that are activated by external stimuli ${ }^{3-6}$. Light, as an external targeting agent, has many unique advantages, including low toxicity, superior spatial and temporal control, and the ability to tune drug activation and/or release by the power, duration and even the wavelength of the light source ${ }^{7-11}$. The utility of light-activated therapies is epitomized by the success of photodynamic therapy (PDT) ${ }^{12-16}$. PDT employs a photosensitizer that, upon exposure to light and oxygen, generates reactive oxygen species (singlet oxygen, hydroxyl radical, peroxides and superoxide $)^{12-15,17}$. The highly reactive, cytotoxic species oxidize, and, thereby, damage, key biomolecules (for example, DNA) critical for cell viability. PDT is clinically employed in dermatology and cancer therapy, and is typically well tolerated by patients ${ }^{12,15}$. Optogenetics is a related technology that employs genetically encoded, light-activated proteins to elucidate the spatial and temporal elements of a variety of biochemical processes, which include mapping the functional neuronal pathways of the brain ${ }^{18}$. Both PDT and optogenetics demonstrate that the physical properties of light, coupled with continued developments in light delivery technology, provide the means to influence biology with exquisite precision. Given the success of PDT and the potential advantages of light-directed therapy, it is surprising that the use of light to trigger the targeted delivery of drugs (phototherapeutics) has yet to find clinical application.

The tissue-penetrating ability of light is dictated by four parameters. First, the nature of the tissue itself plays a key role, since some tissues (for example, the eye) are more transparent to light than others. Second, light delivery parameters (such as laser power and pulsed versus continuous settings) and technology are, likewise, capable of modulating tissue penetrance. Furthermore, where tissue depth is a potential issue, new developments in light delivery technology, such as biodegradable fibre optics ${ }^{19,20}$ and small wireless light sources ${ }^{21}$, serve as alternatives to traditional external light sources. Third, the ability to generate a required photon flux at a specific tissue depth represents only half of the equation. The phototherapeutic also determines whether a specific light intensity is sufficient for a functional response. In particular, the ability of the light-responsive drug to capture photons (extinction coefficient $(\varepsilon)$ ) and subsequently translate photon 
capture into drug action (quantum yield $(\Phi)$ ) are key photophysical parameters. The quantum yield is given by the number of molecules or moles of product formed divided by the number or moles of photons absorbed by the chromophore. Reported $\Phi$ values vary over several orders of magnitude, from $<0.1 \%$ to $30 \%$ and greater ${ }^{22,23}$. The product of $\varepsilon$ and $\Phi(\varepsilon \Phi$ (quantum efficiency)) offers a measure of the light responsiveness of the phototherapeutic. A $\varepsilon \Phi$ value of $\sim 100 \mathrm{M}^{-1} \mathrm{~cm}^{-1}$ is typically benchmarked as good ${ }^{24}$, and a value of $1,000 \mathrm{M}^{-1} \mathrm{~cm}^{-1}$ as excellent ${ }^{25}$, although values in excess of $10,000 \mathrm{M}^{-1} \mathrm{~cm}^{-1}$ have also been reported ${ }^{26}$. In short, both $\varepsilon$ and $\Phi$ of the light-activated drug determine how penetrant light must be to have a functional impact. Fourth, tissue penetrance is dramatically impaired at short wavelengths $(<600 \mathrm{~nm})$, due to the presence of light-absorbing biomolecules (such as haemoglobin and melanin). Until recently, nearly all reported photoactivatable compounds absorb short-wavelength photons (300-450 nm), wavelengths that would otherwise restrict activation to no more than a few millimetres in tissue depth. This limitation is partly responsible for the intense interest in two-photon ${ }^{27,28}$ and upconverting nanoparticle ${ }^{29}$ technologies. However, both technologies have shortcomings with respect to potential clinical applications, which include inefficient release and the requirement for high-powered and specialized lasers ${ }^{29,30}$.

Phototherapeutics that respond to wavelengths within the optical window of tissue (600-900 nm) (FIG. 1) are a relatively recent innovation, with potentially profound clinical ramifications. For example, studies have demonstrated that $800-\mathrm{nm}$ photons display a brain penetrance (through the skull) of up to $3 \mathrm{~cm}$ $\left(\mathrm{REFS}^{31-38}\right)$, thereby, exemplifying potential biomedical opportunities. We note that a large number of reviews have appeared on the general design and properties of photoactivatable compounds, and a few representative comprehensive articles are cited here $\mathrm{e}^{7,39-48}$ and in appropriate places below. This Review focuses on the design of long-wavelength-responsive scaffolds (>600 nm) that mediate drug activation and/or release. In addition, we discuss the prospective path to clinically useful phototherapeutics.

\section{Photoresponsive scaffolds that trigger bond lysis}

Two general design strategies have been used to construct photoactivatable therapeutic agents. Most commonly, an essential functional group on the therapeutic is covalently modified with a photocleavable moiety. Photolysis transforms the biologically inactive 'caged' compound to the active therapeutic. Alternatively, the therapeutic is retained within a carrier by virtue of a photocleavable moiety. This design approach controls the ability of the therapeutic agent to interact with its biological target.

\section{BODIPY scaffolds}

BODIPY, a fluorophore that absorbs green light and is relatively easy to synthesize, is stable in various media, has low toxicity and is an excellent chromophore $\left(\varepsilon=\sim 10^{5} \mathrm{M}^{-1} \mathrm{~cm}^{-1}\right)^{25,49-53}$. Several research groups have developed photocleavable scaffolds using a meso-methyl BODIPY (boron-dipyrromethene) core. The meso-methyl BODIPY group (1) has been used to mask several functional groups, including carboxylic acids and alcohols. The mechanism of photorelease (green light) is hypothesized to proceed via an $\mathrm{S}_{\mathrm{N}} 1$ reaction through a carbocation intermediate, followed by the nucleophilic attack of the solvent ${ }^{50,52,53}$ (FIG. 2). Subsequent studies were conducted to optimize this BODIPY scaffold and found that the addition of a heavy atom, such as Br or I, and boron alkylation improves the quantum yield of photorelease by up to two orders of magnitude ${ }^{25,50,54}$. Furthermore, the nature of the leaving group also influences photolytic efficiency, which

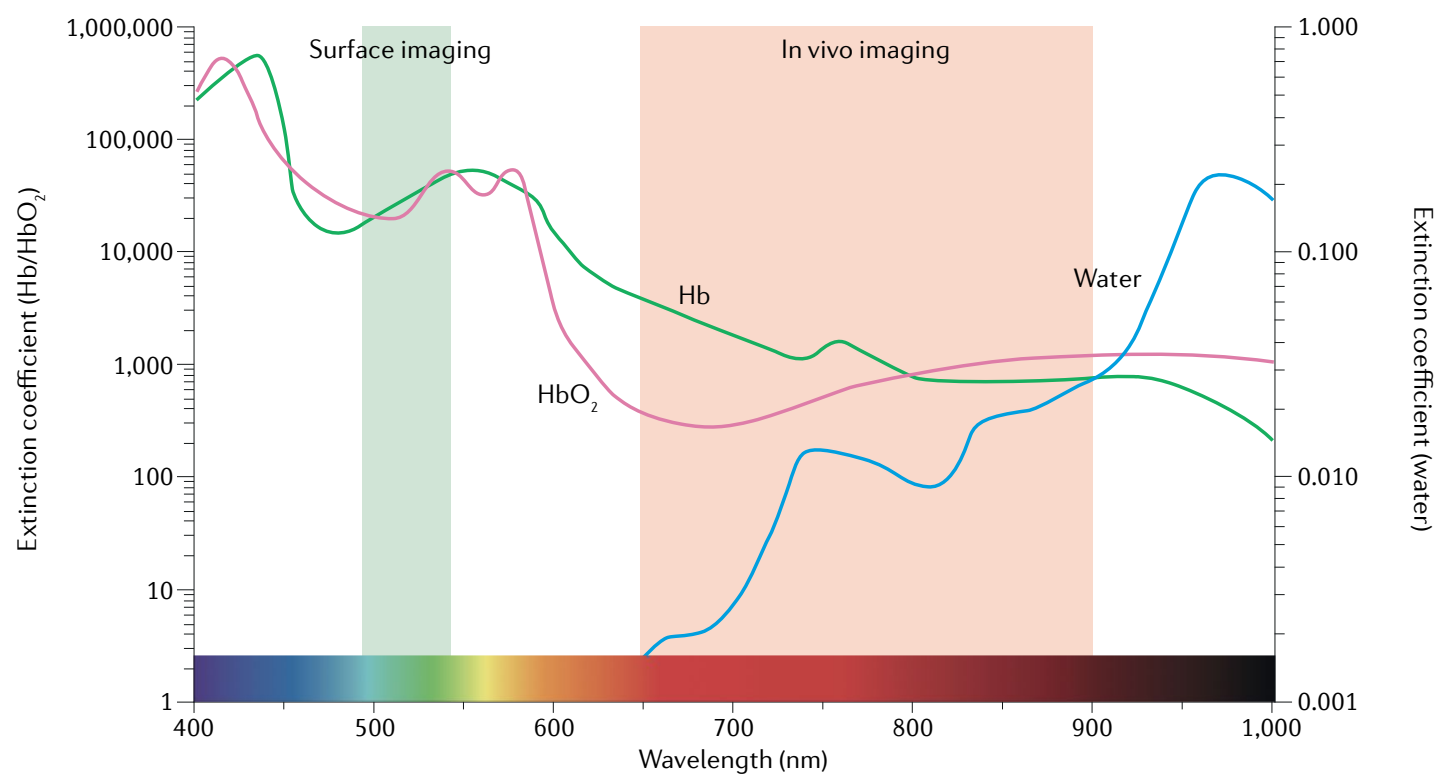

Fig. 1 | The extinction coefficient as a function of wavelength for $\mathrm{Hb}, \mathrm{HbO}_{2}$ and water. The region from 600 to $900 \mathrm{~nm}$ has been referred to as the optical window of tissue due to enhanced tissue penetrance at these wavelengths. $\mathrm{Hb}$, haemoglobin; $\mathrm{HbO}_{2}$, oxygenated haemoglobin. Adapted with permission from REF. ${ }^{147}$, American Chemical Society. 


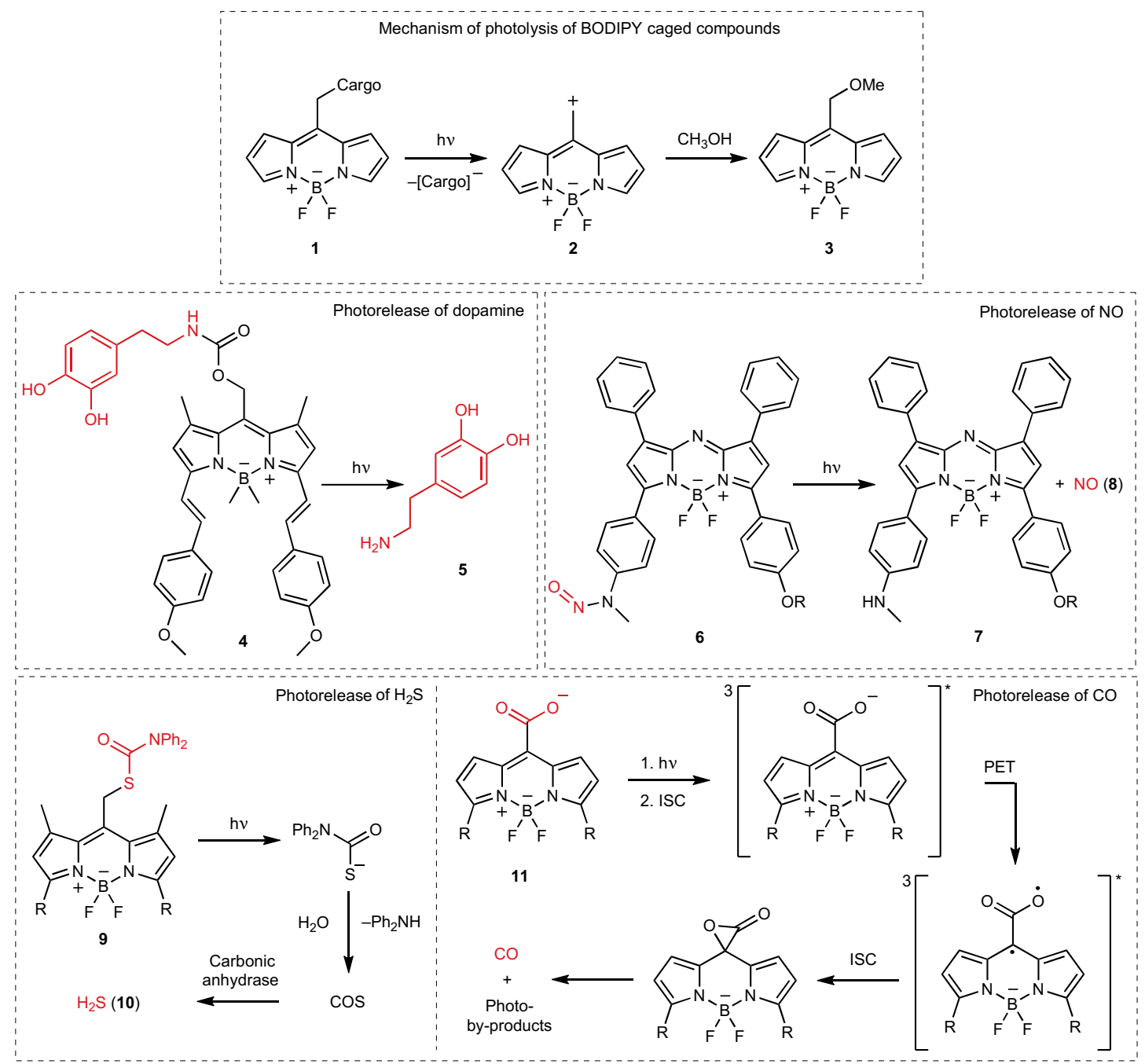

Fig. 2 | Photolysis of BODIPY caged compounds. Light-triggered BODIPY uncaging has been demonstrated to release several compounds. It generally proceeds via an $\mathrm{S}_{\mathrm{N}} 1$ mechanism (1-3), where it can release dopamine (5), $\mathrm{NO}(\mathbf{8})$ and $\mathrm{H}_{2} \mathrm{~S}(\mathbf{1 0})$. Conversely, CO formation transpires through a photoinduced electron transfer (PET) step and intersystem crossing (ISC).

clearly has implications in the caging of various drugs $s^{50}$. With these features in mind, a collection of BODIPY derivatives with extended conjugation $(660-690 \mathrm{~nm})$ have been described and used to cage and photorelease various carboxylic acid derivatives ${ }^{25}$ (FIC. 2).

Amine-containing derivatives have also been photodeprotected, as exemplified by the dopamine derivative $4\left(\mathrm{REF}^{49}{ }^{4}\right.$ ) (FIG. 2). Dopamine (5) is not only an important neurotransmitter but it also plays a key role in the regulation of cardiac function by increasing heart rate and contractile force. Indeed, exposure of cultured cardiomyocytes to the 652-nm photolyzed BODIPY-protected dopamine elicits an enhanced beating frequency comparable with that of cardiomyocytes treated with pure dopamine. These investigators also performed key control experiments, namely, studies in the absence of illumination, which are essential for validating light-dependent activity. The gold standard of a phototherapeutic is the absence of biological activity in the dark, since undesired dark activity in vivo raises the prospect of metabolic conversion of the caged prodrug to its active counterpart. With respect to the
BODIPY-protected dopamine derivative, in the absence of photolysis, a slightly higher basal beating frequency is observed relative to untreated cardiomyocytes ${ }^{49}$. However, the slight dark activity displayed by caged dopamine is significantly less than that of its photolyzed counterpart.

The BODIPY scaffold has also been used to trigger and monitor the release of nitric oxide (NO) in a mouse model $^{55}$. NO is a gasotransmitter that participates in neurotransmission, vasodilatation, hormone secretion and wound repair ${ }^{56}$. Given the biochemical promiscuity of NO, using light to direct NO release has received significant attention. Illumination of the aza-BODIPY scaffold (6, where $\mathrm{R}=\mathrm{CH}_{3}$ or the more water-soluble $\mathrm{R}=$ trimethylammonium derivative) results in photolysis of the $\mathrm{N}$-nitroso bonds and generation of NO (REF. ${ }^{55}$ ) (FIG. 2). Due to the loss of the electron-withdrawing $\mathrm{N}$-nitroso group during photolysis, the by-product (7) is bathochromically shifted by $70 \mathrm{~nm}$ relative to the starting material ${ }^{55}$. Consequently, two wavelengths $\left(\lambda_{\text {blue }}\right.$ and $\lambda_{\text {red }}$ ) can be used to monitor the amount of starting material (6) and product (7) present, which was 
demonstrated in vivo using a tumour mouse model (see the section entitled The phototherapeutic effect).

Hydrogen sulfide $\left(\mathrm{H}_{2} \mathrm{~S}\right)$ is another gasotransmitter that elicits antioxidative, anti-inflammatory and other cytoprotective effects ${ }^{57,58}$. Like NO, the targeted photorelease of $\mathrm{H}_{2} \mathrm{~S}$ has potential therapeutic implications, particularly in the areas of cardiovascular disease, obesity, diabetes, ageing and neurodegenerative diseases ${ }^{58-61}$. A red-shifted BODIPY derivative $9(\mathrm{R}=$ styryl moiety) possesses an $S$-thiocarbamate moiety that serves as the incipient $\mathrm{H}_{2} \mathrm{~S}$ (FIG. 2). However, photorelease does not directly furnish the gasotransmitter but, rather, gaseous carbonyl sulfide. The latter is enzymatically converted to $\mathrm{H}_{2} \mathrm{~S}$ via carbonic anhydrase, a ubiquitous enzyme found in mammalian tissues. The photogeneration $(700 \mathrm{~nm})$ of $\mathrm{H}_{2} \mathrm{~S}$ from 9 was confirmed in a human liver cancer cell line (HepG2) using a fluorogenic $\mathrm{H}_{2} \mathrm{~S}$ probe (SF7-AM).

Light-responsive carbon monoxide (CO)-releasing molecules (photoCORMs) have potential therapeutic implications for inflammatory, vascular and oncogenic diseases $^{22,62}$. However, CO is infamous for its ability to bind to haemoglobin 200-fold more strongly than oxygen, with lethal consequences at high concentrations. Therefore, precise delivery of the right amount of $\mathrm{CO}$ at the specific region of interest is crucial ${ }^{63}$. Most photoCORMs are transition-metal-based and respond to wavelengths outside of the optical window of tissue. In contrast, the BODIPY scaffold that was used to create the first photoCORM (11) absorbs well into the far red $\left(\lambda_{\max }=652 \mathrm{~nm}\right.$, but with the absorption band out to $730 \mathrm{~nm})^{22}$. The proposed mechanism of CO release involves intersystem crossing and photoinduced electron transfer steps (FIG. 2). In vivo studies with 11 confirmed photo-triggered release of CO.

Scope and opportunities. The BODIPY scaffold contains a number of exemplary features, including excellent $\varepsilon$ and $\Phi$ values, as well as maximal wavelengths of photoactivation (well into the $750+\mathrm{nm}$ range). A limited number of bioactive species has been caged using BODIPY derivatives, primarily gasotransmitters. Although the latter have been studied in vivo, detailed analyses on the metabolic stability of BODIPY caged derivatives remain to be conducted. Finally, as discussed in a later section, the incorporation of long-wavelengthresponsive BODIPY derivatives into carrier-based constructs represents a potential opportunity for the photo-delivery of an assortment of therapeutic agents.

\section{Cyanine scaffolds}

Another fluorophore scaffold that holds promise as a photon-absorbing agent for caging bioactive compounds belongs to the cyanine family of fluorophores ${ }^{64,65}$. Heptamethine cyanines have been used in a variety of bioimaging applications, and some derivatives are well-tolerated in humans ${ }^{66-68}$. Cyanine derivatives absorb far-red light, have large $\varepsilon$ values and are detected even under demanding in vivo conditions ${ }^{66-69}$. A cyanine-flavonol conjugate has been described with excellent optical properties (photolysis at $800 \mathrm{~nm}$ with $\varepsilon=250,000 \mathrm{M}^{-1} \mathrm{~cm}^{-1}$ ), capable of efficiently releasing two CO molecules per CORM 12 (FIG. 3) (110-fold greater than 11) ${ }^{68}$. Mechanistic studies confirmed that the source of $\mathrm{CO}$ is the flavonol, as revealed by the photo-by-product (13). Cell-based and in vivo studies were also conducted with $\mathbf{1 2}$, which demonstrated light-dependent CO release ${ }^{68}$.

The utility of the heptamethine cyanine scaffold as a near-infrared photocaging group was examined for phenol-containing therapeutics ${ }^{66}$. The premise of this strategy is based on light-induced photooxidative cleavage at the $\mathrm{C} 1-\mathrm{Cl}^{\prime}$ and/or $\mathrm{C}^{\prime}-\mathrm{C}^{\prime}$ bonds of the cyanine $\pi$ system (14), thereby, rendering the $C 4^{\prime}-\mathrm{N}$ bond susceptible to hydrolysis. The resultant free amine subsequently promotes an intramolecular cyclization to yield the parent drug ${ }^{66,67,69}$ (FIC. 3). A library of cyanine derivatives was prepared to study the structure-function relationships of various photorelease properties, including $\lambda_{\max }$, photooxidation efficiency, uncaging kinetics and dark hydrolysis rate ${ }^{69}$. The lead derivative (18) contains an $N, N^{\prime}$-diethylethylenediamine linker between the chromophore scaffold and the site that houses the drug cargo. Compound 18 displays a $\lambda_{\text {ex }}$ in the far red $(732 \mathrm{~nm})$ and minimal dark hydrolysis $(10 \%$ after 10 days). With the lead chromophore in hand, the light-triggered drug delivery design strategy was tested in vivo using an antibody-drug conjugate (ADC) motif. Although ADCs do contain a disease-targeting antibody moiety, the release of the attached drug in a controlled spatiotemporal manner remains a challenge ${ }^{67,69}$. The alkyne moiety on $\mathbf{1 8}$ was employed to covalently attach the exceptionally cytotoxic alkylating agent duocarmycin (19) to the antibody of choice. This ADC phototherapeutic had been used to site-selectively deliver duocarmycin in a tumour-bearing mouse model (see the section entitled The phototherapeutic effect).

The heptamethine cyanine chromophore has, likewise, been employed to construct the caged cisplatin derivative $\mathbf{2 0}\left(\mathrm{REF}^{2}{ }^{23}\right)$. The promiscuity of Pt-based chemotherapeutics often results in undesired systemic toxicity and limits the dosage that can be given to patients. Consequently, spatially controlled generation of the aquated/active form could reduce toxicity. Although a number of light-promoted Pt complexes have been described $^{70}$, this work is the first example of activation in the optical window of tissue (at $790 \mathrm{~nm}$ ). In addition to photorelease of the bioactive platin species, these investigators demonstrated that the cyanine dye exerts a photodynamic effect, thereby, simultaneously generating reactive oxygen species. There is a noteworthy element to this observation that has consequences well beyond this particular study. Nearly all of the chromophores described in this Review are long-wavelength fluorophores, many of which can serve as photodynamic agents. Therefore, it is essential that control experiments are designed to properly attribute the observed biological response to the mechanism of action (that is, drug release versus the generation of reactive oxygen species).

Scope and opportunities. The cyanine scaffold possesses an extraordinarily large $\varepsilon$, as well as the demonstrated release of chemotherapeutic agents. It will be interesting to see if this strategy can be extended to a variety of wavelengths. As with the BODIPY caged gasotransmitters, 


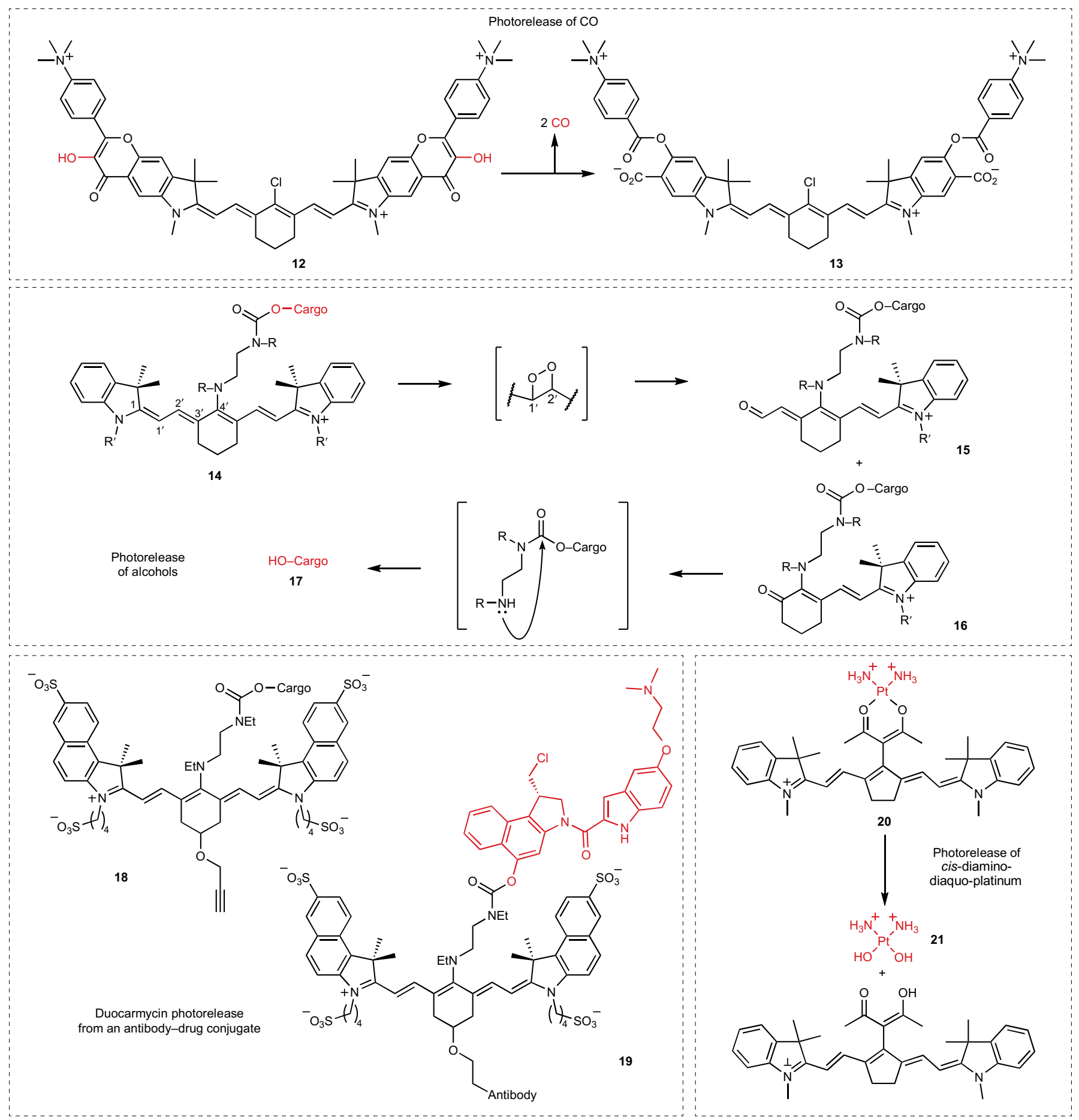

Fig. 3 | Photorelease of compounds from cyanines. Cyanines have been used to photorelease CO from 12, alcoholcontaining cargo from 14 (for example, duocarmycin from an antibody-drug conjugate (19)) and cis-diamino-diaquoplatinum from 20. The acetyleneic cyanine derivative $\mathbf{1 8}$ can be coupled to antibodies to create light-responsive antibody-drug conjugates. Drug components are highlighted in red.

site-directed photorelease has been demonstrated in vivo with 19. For the latter, the mechanism of release requires oxygen. Although not explicitly tested, release of the active form of cis-platin from $\mathbf{2 0}$ presumably transpires in the absence of oxygen. Oxygen dependency limits the utility of this scaffold in hypoxic settings, such as the tumour microenvironment. However, an analogue of the cyanine scaffold has recently been described that operates independently of oxygen by employing a photoinduced electron transfer drug release mechanism ${ }^{71}$. Finally, the incorporation of a cyanine-based caged chemotherapeutic onto an antibody ${ }^{67}$ and in micelles ${ }^{71}$ demonstrates the feasibility of using this chromophore in carrier-based constructs. 


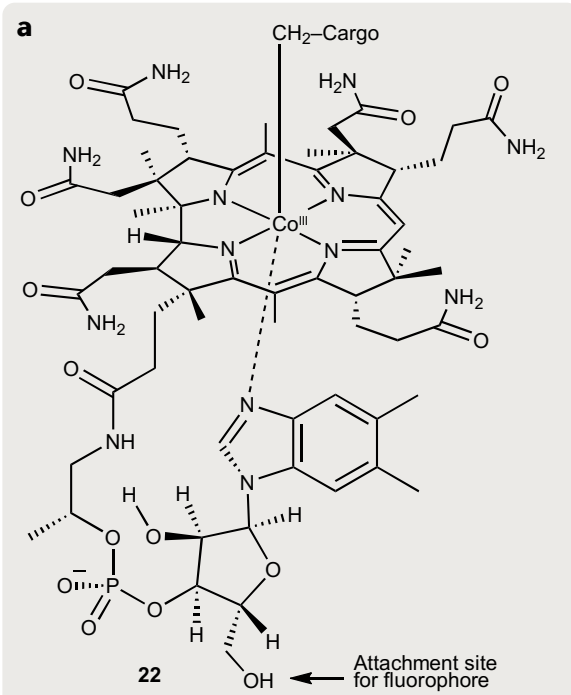

b Proposed PET photolysis mechanism for drug-Cbl-fluorophore derivatives
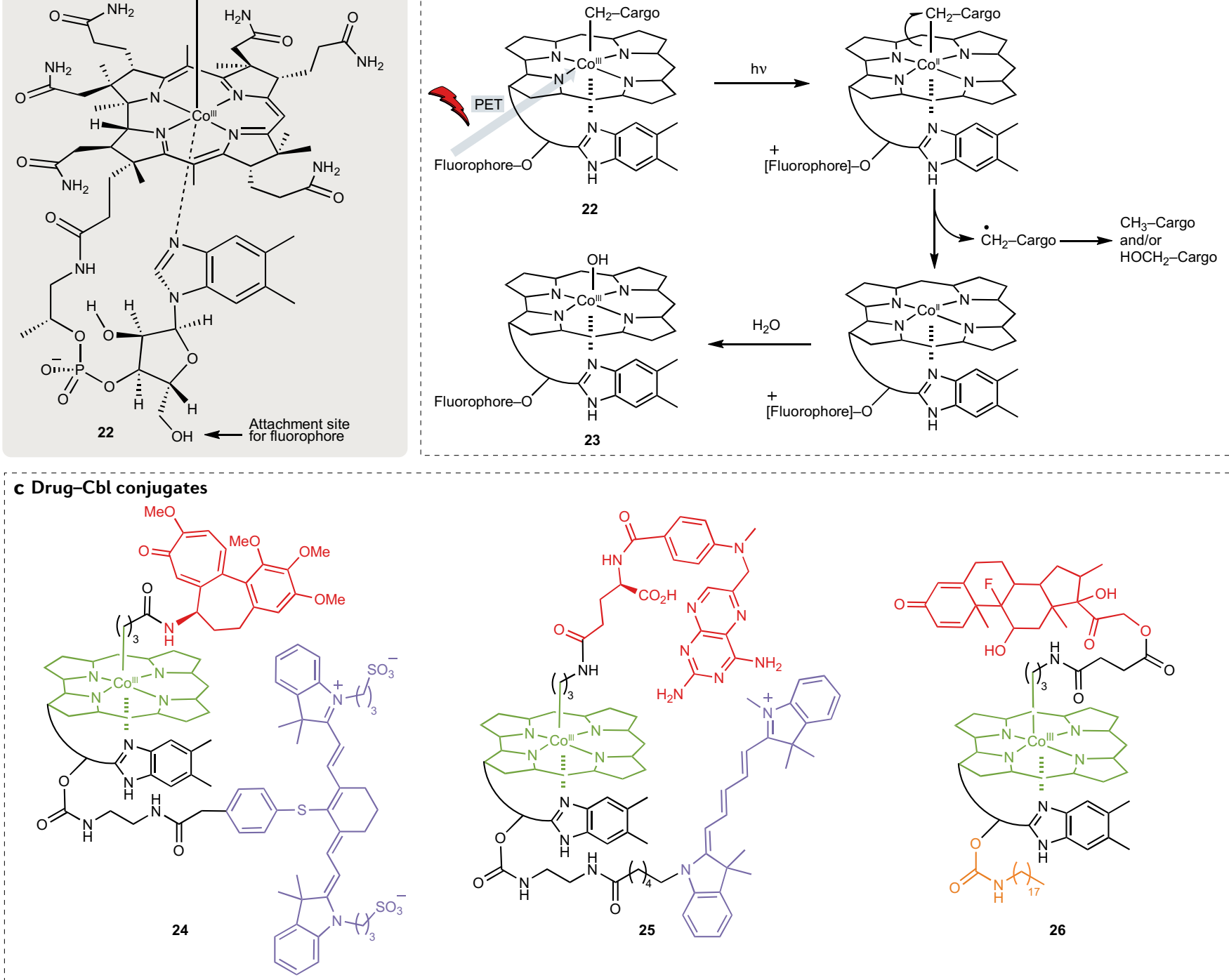

26
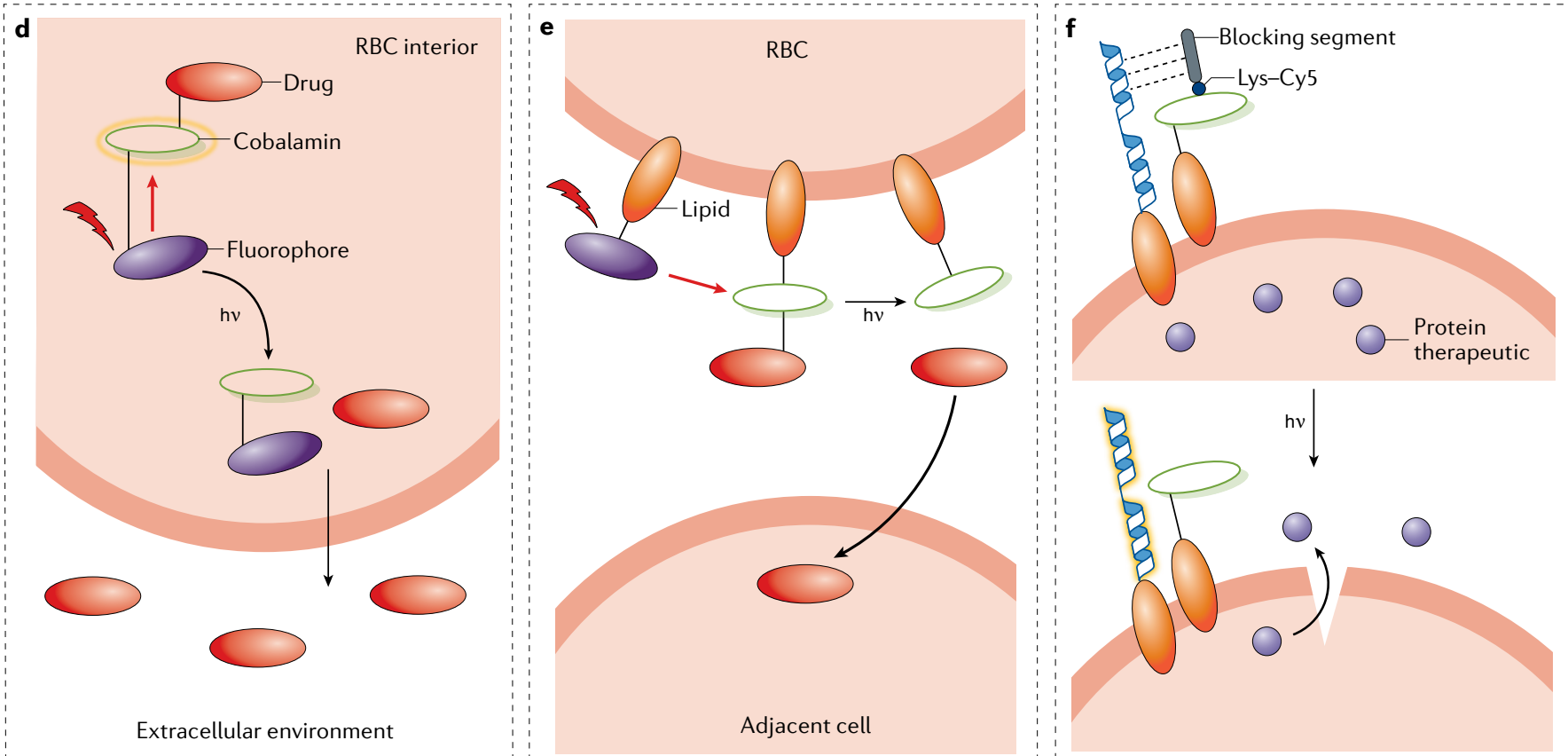
4 Fig. 4 | Photolysis of alkyl-cobalamin-fluorophore caged compounds. a | Full structure of a 'cargo' substituted alkyl-cobalamin (alkyl-Cbl) (22). b | Schematic structures of alkyl-Cbl-fluorophores. Excitation of the appended fluorophore (22) is presumed to trigger a photoinduced electron transfer (PET) that either reduces (shown) or oxidizes Co'II, which results in the subsequent release of the $-\mathrm{CH}_{2} \mathrm{R}$ moiety and formation of the hydroxy-Cbl by-product (23). c| Example drug-Cbl-fluorophore conjugates $(\mathbf{2 4 , 2 5}$ ). The conjugates are designed to execute the mechanism shown in parts $\mathbf{d}$, e or $\mathbf{f}$. $\mathbf{d}$ |The conjugates are loaded into the interior of red blood cells (RBCs) by pore-forming hypotonic conditions. Subsequent restoration of isotonicity seals the pore and traps the phototherapeutic in the RBC. Photolysis frees the drug from the membrane-impermeable Cbl. e | Lipidated drug-Cbl conjugates (26) and lipidated fluorophores self-assemble onto the surface of RBCs, which forms an active phototherapeutic array. Illumination releases the drug from the Cbl-lipid anchor. $\mathbf{f} \mid$ Design of a photohaemolytic RBC. The engineered RBC is internally loaded with protein therapeutic and externally modified with a photohaemolytic trigger comprised of melittin (Mel; blue helix) and a blocking segment peptide that inhibits Mel haemolytic activity. Photolysis releases the blocking segment peptide, which activates Mel and subsequently releases the therapeutic proteins. Part $\mathbf{d}$ adapted with permission from REF. ${ }^{80}$, Wiley. Part e adapted with permission from REF. ${ }^{78}$, Wiley. Part $\mathbf{f}$ adapted with permission from REF. ${ }^{85}$, American Chemical Society.

\section{Cobalamin scaffolds}

The Co ${ }^{\mathrm{III}}-\mathrm{C}$ bond of alkyl-cobalamins (alkyl-Cbl) (22) has long been known to undergo homolytic cleavage upon absorption of light within the 330-575-nm range $^{72-74}$. In at least one case, this transpires with an astounding initial quantum yield $(\Phi=1)^{74}$. However, efficient photogeneration of the $\mathrm{Co}^{\mathrm{II}}$ and $\mathrm{R} \cdot$ intermediates is offset by rapid recombination. Nonetheless, it is not uncommon to obtain final $\Phi$ values of $0.2-0.5$, exceptional values in the photochemical realm. In contrast to the BODIPY derivatives, extension of alkyl-Cbl photochemistry into the red, far-red and near-infrared wavelengths was not achieved by manipulating the $\pi$ system of the chromophore. Instead, long-wavelength fluorophores were installed adjacent to the corrin ring (for example, on the ribose moiety $)^{72}$. Excitation of the fluorophore is presumed to trigger an electron transfer, resulting in the formation of exceptionally unstable $\mathrm{Co}^{\mathrm{II}}-\mathrm{R}$ and/or $\mathrm{Co}^{\mathrm{IV}}-\mathrm{R}$ bonds ${ }^{75-77}$, and the subsequent release of $\mathrm{R}^{\cdot}$ and/or $\mathrm{R}^{+}$, respectively (FIG. 4). The modular nature of this strategy allows the wavelength of photocleavage to be pre-assigned by choosing from a wide variety of commercially available fluorophores $(600-800 \mathrm{~nm})$. Furthermore, designated photolysis wavelengths enables the construction of orthogonally photoresponsive compounds that can be distinguished on the basis of wavelength alone ${ }^{78-81}$.

An array of small molecule drugs, including colchicine (24), methotrexate (25), paclitaxel and docetaxel, have been attached to Co via amine-containing or carboxylic-acid-containing linkers ${ }^{79,80}$. However, unlike traditional caged molecules, the site chosen for drug modification does not interfere with drug activity. Rather, the Cbl moiety is known to be membraneimpermeable, and this property was used to entrap drug-Cbl-fluorophore conjugates within red blood cells (RBCs). Specifically, drug-Cbl-fluorophore derivatives are readily loaded into RBCs under hypotonic conditions, resulting in swelling and pore formation in the cell membrane. Subsequent restoration of isotonicity reseals the pores and traps the drug-Cbl-fluorophore conjugate in the RBC interior ${ }^{79,80}$ (FIG. 4). Upon exposure to the appropriate wavelength of light (for example, $\mathbf{2 4}$ at $780 \mathrm{~nm}, 25$ at $650 \mathrm{~nm}$ ), the Co-C bond is photolyzed and the now membrane-permeable drug escapes from the RBC. Concentrations as high as $40 \mu \mathrm{M}$ drug-Cblfluorophore have been loaded into RBCs. RBCs containing a photoresponsive taxol derivative were introduced into mice (via tail vein injection) and photoreleased with well-defined spatiotemporal control ${ }^{79}$ (see the section entitled The phototherapeutic effect). By contrast, the inactive control derivative $\left(\mathrm{H}_{2} \mathrm{O}-\mathrm{Cbl}\right.$-fluorophore) loaded into RBCs was not biologically active.

The strategy outlined in FIG. $4 \mathrm{~d}$ requires the therapeutic to readily diffuse through cell membranes. An alternative approach has been developed for membraneimpermeable agents, such as peptide-based drugs ${ }^{82}$. The Cbl-drug conjugate is appended to the RBC surface using a lipid anchor, as illustrated for the dexamethasone conjugate 26. Long-wavelength antennas, in the form of lipidated fluorophores, can be simultaneously anchored to the surface of the RBCs as well ${ }^{78}$ (FIG. 4 e). As in the case of internally loaded Cbl-drug conjugates (FIG. 4d), a variety of drugs and fluorophores $\left(\lambda_{\mathrm{ex}}=600-800 \mathrm{~nm}\right)$ has been used to photorelease therapeutic agents from the surface of $\mathrm{RBCs}^{78}$.

Protein therapeutics is another class of drugs that could benefit from targeted delivery mechanisms ${ }^{83,84}$. The RBC-conveyed drug delivery strategy has been expanded to include protein therapeutics using a photohaemolytic trigger comprised of melittin (Mel), an active component of bee venom ${ }^{85}$ (FIG. 4f). Although Mel has received significant attention as an anticancer, anti-inflammatory and antimicrobial agent, it is perhaps best known for its ability to bind to and lyse RBCs. RBCs are first loaded with the therapeutic protein of interest using the hypotonic loading method described above. Following RBC pore resealing, which results in protein entrapment, the $\mathrm{RBC}$ surface is subsequently exposed to $\mathrm{C}_{18}-\mathrm{Mel}$ and a lipidated $\mathrm{Cbl}$ derivative covalently attached to a 'blocking segment' (BS) peptide $\left(\mathrm{C}_{18}-\mathrm{Cbl}-\mathrm{BS}\right)$. The BS peptide interacts with Mel and inhibits its lytic activity. Upon illumination, the Co-C bond is photolyzed, the BS peptide is released from the RBC surface, Mel-induced haemolytic activity is unleashed and the internally loaded therapeutic protein is released. Furthermore, the wavelength of photorelease is assigned by attaching a fluorophore with the desired excitation profile to the BS peptide $\left(\mathrm{C}_{18}-\mathrm{Cbl}-\mathrm{Cy} 5-\mathrm{BS}\right)$, as evidenced by photohaemolysis at $650 \mathrm{~nm}$ using the $\mathrm{Cy} 5$ fluorophore (FIG. $4 \mathrm{f}$ ). Proteins loaded and photoreleased include the vascular endothelial growth factor (VEGF), which has received significant attention for therapeutic angiogenesis ${ }^{86}$, and thrombin (see the section entitled The phototherapeutic effect), which is representative of clotting proteins ${ }^{85}$.

Scope and opportunities. The Cbl scaffold has been applied to a wide variety of drugs, including small molecules, peptides and proteins. Furthermore, the wavelength of activation is readily set using very bright, commercially available fluorophores. Large $\varepsilon$ in conjunction with excellent $\Phi$ suggests that Cbl-based phototherapeutics should display good quantum efficiency under in vivo conditions. However, a key limitation is that the freed drug molecule is conjugated to a 


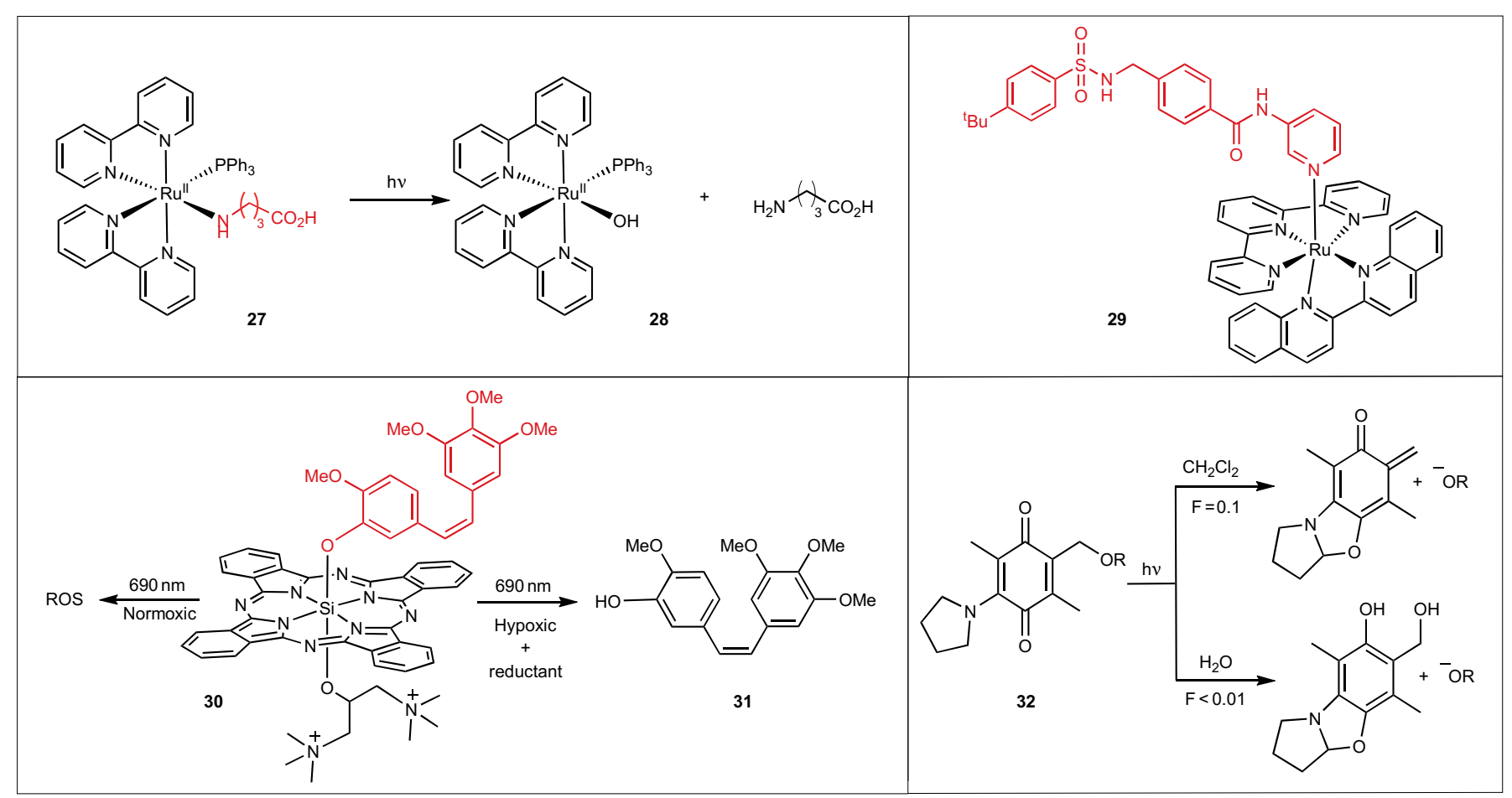

Fig. 5 | Photolysis of Ru", silicon phthalocyanine and amino-1,4-benzoquinone scaffolds. Photolysis of the Ru-bipyridyl complex $\mathbf{2 7}$ furnishes $\gamma$-aminobutyric acid. The pyridyl-containing species $\mathbf{2 9}$ likewise undergoes photolysis. The silicon phthalocyanine scaffold (30) generates reactive oxygen species (ROS) under normal oxygen conditions but suffers Si-O bond cleavage under hypoxic conditions in the presence of reducing agents. The yield of the amino-1,4-benzoquinone scaffold 32 photolysis product is quenched under aqueous conditions. However, water-soluble nanoparticles can be used to overcome this limitation.

small linker. This could complicate regulatory approval. By contrast, the photo-rupture of RBCs (FIG. 4f) avoids this potential problem, at least for proteins and other membrane-impermeable drugs.

\section{$R u^{\prime \prime}$ scaffolds}

Ruthenium $\left(\mathrm{Ru}^{\mathrm{II}}\right)$-based bipyridine derivatives were first introduced as caging agents for nitrogen-containing neurotransmitters ${ }^{87-91}$. Although these derivatives proved to be effective light-activatable agents for in vitro biological studies, their instability in the dark, low $\varepsilon$ and poor $\Phi(0.001-0.1)$, and maximum absorption $(\lambda<500 \mathrm{~nm})$ outside of the optical window of tissue are less than ideal for in vivo applications ${ }^{92}$. However, $\Phi$ values can be improved by an order of magnitude through the simple introduction of a triphenylphosphine ligand (27) $)^{91}$ (FIG. 5). Bathochromically shifted ruthenium complexes have been reported that release the cytochrome P450 inhibitor abiraterone ${ }^{93}$. In addition, a red-light-activated ruthenium-caged inhibitor of nicotinamide phosphoribosyltransferase has been prepared and characterized $\left(29 ; \lambda_{\max }=531 \mathrm{~nm}\right.$, but photoresponsive out to $625 \mathrm{~nm})^{94}$. Finally, we note that ruthenium complexes not only coordinate and photorelease primary amines (for example, 27) and aromatic nitrogen-containing compounds (for example, 29) but nitrile-containing ${ }^{95}$ and thioether-containing ${ }^{96}$ compounds as well. For example, nitrile ligands undergo photodissociation with near-infrared light irradiation $\left(\lambda_{\max }\right.$ in $\mathrm{H}_{2} \mathrm{O}=497-517 \mathrm{~nm}$, but photoresponsive out to
$655 \mathrm{~nm}$ ) from $\mathrm{Ru}$-terpyridine compounds containing electron-donating acetylacetonate groups ${ }^{97,98}$.

The effect of electronic and steric factors on key photophysical properties has been described for a variety of previously developed $\mathrm{Ru}^{\mathrm{II}}$ complexes ${ }^{92}$. As expected, bidentate ligands with extended $\pi$ systems generate bathochromically shifted complexes $\left(\lambda_{\max }\right.$ values out to the mid-500s, with tails out to the mid-600s). Furthermore, the structural nature of the photoreleasable ligand has a significant impact on the stability and the $\Phi$ of the complex. Finally, an increase in the $\Phi$ was found to correlate with a decrease in the overall dark stability of the compound. However, derivatives were identified with favourable dark stability and photophysical properties that offer promise for this intriguing class of photoresponsive scaffolds.

Scope and opportunities. The $\mathrm{Ru}^{\mathrm{II}}$-based scaffold benefits from certain unique properties not seen with other light-responsive scaffolds, particularly the ability to coordinate and photorelease pyridine-containing and nitrile-containing compounds. However, extension of the absorption of $\mathrm{Ru}^{\mathrm{II}}$ complexes into the far-red region remains a work in progress. The $\varepsilon$ values for red light photolysis are modest, which suggests, as currently devised, that the use of these derivatives for deep tissue applications is not ideal. Finally, as noted by others, in vivo studies of light-responsive Ru-based complexes are limited ${ }^{99}$. Consequently, a detailed examination of the stability, toxicity and efficacy of these complexes in 
animal models of human disease are needed to assess their potential in the clinic.

\section{Prospective photolytic scaffolds}

The photoresponsive moieties described in the previous sections have received significant attention as scaffolding for the construction of phototherapeutics. This section will focus on two promising long-wavelength-responsive protecting groups that have been recently employed for light-activated drug release.

The silicon phthalocyanine ( $\mathrm{SiPc}$ ) scaffold possess a photolabile $\mathrm{Si}-\mathrm{O}$ bond that is responsive to $690-\mathrm{nm}$ light $^{100}$ (FIC. 5). The photochemistry of the SiPc scaffold was explored with a SiPc-combretastatin-A4 conjugate containing a water-solubilizing 1,3-bis(trimethylaminium)2-propanol ligand (30). Under normal oxygen conditions, the $\Phi$ for ${ }^{1} \mathrm{O}_{2}$ generation is excellent, at 0.39 . By contrast, the quantum yield for uncaging (formation of 31) under these conditions is negligible at $3 \times 10^{-6}$. The latter improves by 900 -fold in the absence of oxygen $\left(\Phi=2.7 \times 10^{-3}\right)^{100}$. In short, at present, the SiPc scaffold is limited to uncaging of phenols and only modestly transpires under hypoxic conditions. In addition, the SiPc macrocycle by-product is insoluble in aqueous solution, a potentially serious impediment for in vivo applications. However, water-soluble carriers, such as liposomes, could overcome this challenge (see the section entitled Path to clinical applications).

Another potential photolytic scaffold is aminobenzoquinone (32), which absorbs light in the red region (for example, $\left.\varepsilon=1,000 \mathrm{M}^{-1} \mathrm{~cm}^{-1} @ 600 \mathrm{~nm}\right)$ and undergoes photolysis in dichloromethane $(\Phi=0.1)$. Unfortunately, this scaffold's photolytic efficiency is unfavourably impacted by aqueous conditions ( $\Phi<0.01$ in 30\% aqueous $\left.\mathrm{CH}_{3} \mathrm{CN}\right)^{101-103}$. To overcome this limitation, several drug-nanoparticle conjugates have been synthesized, where the caged drug can be embedded within a hydrophobic environment ${ }^{102}$. More recently, the aminobenzoquinone framework has been modified to improve water solubility and quantum yield; however, extension out into the red remains modest $\left(\lambda_{\max }\right.$ at $550 \mathrm{~nm}$ with $\left.\varepsilon=2,500 \mathrm{M}^{-1} \mathrm{~cm}^{-1}\right)$, with a tail out to $\sim 625 \mathrm{~nm}\left(\right.$ REFS $\left.^{104,105}\right)$. The photophysical properties of these derivatives limit their application to easily light-accessible tissues (for example, the skin) or regions where fibre optics can be placed. Furthermore, it is likely that the benzoquinone is susceptible to metabolic reduction and/or nucleophilic attack under in vivo (intracellular) conditions. Nonetheless, given the simplicity of the benzoquinone moiety, it may be possible to generate long-wavelength derivatives with photophysical properties that are more optimally tuned to deep tissue applications.

\section{Photoresponsive scaffolds that trigger bond isomerization}

Photoisomerization represents a potentially attractive alternative to photolysis as a means for controlling biological activity ${ }^{40,106-108}$. This approach involves the interconversion of the cis and trans configurational isomers of a double bond and/or the electrocyclization of trienes. These 'photoswitches' include azobenzenes, spiropyrans, hexaarylbiimidazoles, donor-acceptor Stenhouse adducts (DASAs), hydrazones, indigoids ${ }^{109}$ and other chromophores ${ }^{106,108}$. The overwhelming majority of these species respond to ultraviolet and/or short visible wavelengths. In recent years, a few derivatives have been described that respond to red light.

\section{Azobenzene scaffolds}

The most commonly used chromophore for photoswitches is the azobenzene scaffold $\mathrm{d}^{40,110-112}$. Azobenzenes have been used as photoswitches in a wide variety of molecules, including peptides, proteins, nucleic acids, lipids and carbohydrates ${ }^{40}$. Key features of the azobenzene scaffold are their resistance to photobleaching, good quantum yields, reversibility and a robust literature on the synthesis and properties of azobenzene derivatives. For simple azobenzenes (33), the most thermodynamically stable trans isomer is converted to its cis counterpart in the ultraviolet range (320-340 nm) and the less energetically favourable cis isomer is transformed to its trans counterpart by visible light or thermally (FIG. 6). However, given the therapeutic limitations of short wavelengths, there is intense interest in generating longer-wavelength-responsive azobenzene analogues $^{110,113-115}$.

Although several azobenzene photoswitches have been described that respond to red light $(625 \mathrm{~nm})$, excitation lies at the tail end of the absorption spectra. Consequently, the $\varepsilon$ values in this region are extremely small. Nonetheless, activation by red light is promising, since this suggests that azobenzene derivatives with $\lambda_{\max }$ values in the red have the potential to be invaluable for drug delivery purposes. Indeed, the continued introduction of azobenzene photoswitches with more favourable photophysical properties bodes well for these scaffolds ${ }^{116}$. A potentially useful feature of red-shifted azobenzenes is their tendency to undergo rapid cis to trans dark (thermal) conversions (often on the time frame of seconds at room temperature $)^{40}$. Under these circumstances, it is preferable to have the cis isomer serve as the light-triggered bioactive species (exceptions to this 'rule' have been reported) $)^{117,118}$. The short lifespan of red-shifted cis isomers represents an interesting biomedical opportunity: only species within the illuminated region (that is, at the diseased site) are bioactive; those that diffuse from this area will rapidly reset to the inactive state. However, this may necessitate constant illumination at the site of interest. Clearly, the optimal lifespan for any given bioactive agent remains to be determined when employed in this fashion. Fortunately, tuning cis thermal stability is straightforward via manipulation of substituents on the aromatic ring that are ortho to the azo bridge ${ }^{110}$.

One of the few examples of an azobenzene-based, red-light-triggered activation of a drug employed the antibiotic trimethoprim (34) $)^{119}$. Since many bacterial infections prefer anatomical sites of infestation, it has been argued that spatially targeted phototherapy would use smaller amounts of medicinal agent, mitigate side effects and reduce drug resistance ${ }^{120}$. A library of trimethoprim derivatives (35) was synthesized and screened in order to identify derivatives that display antibacterial activity with a pronounced dependence 

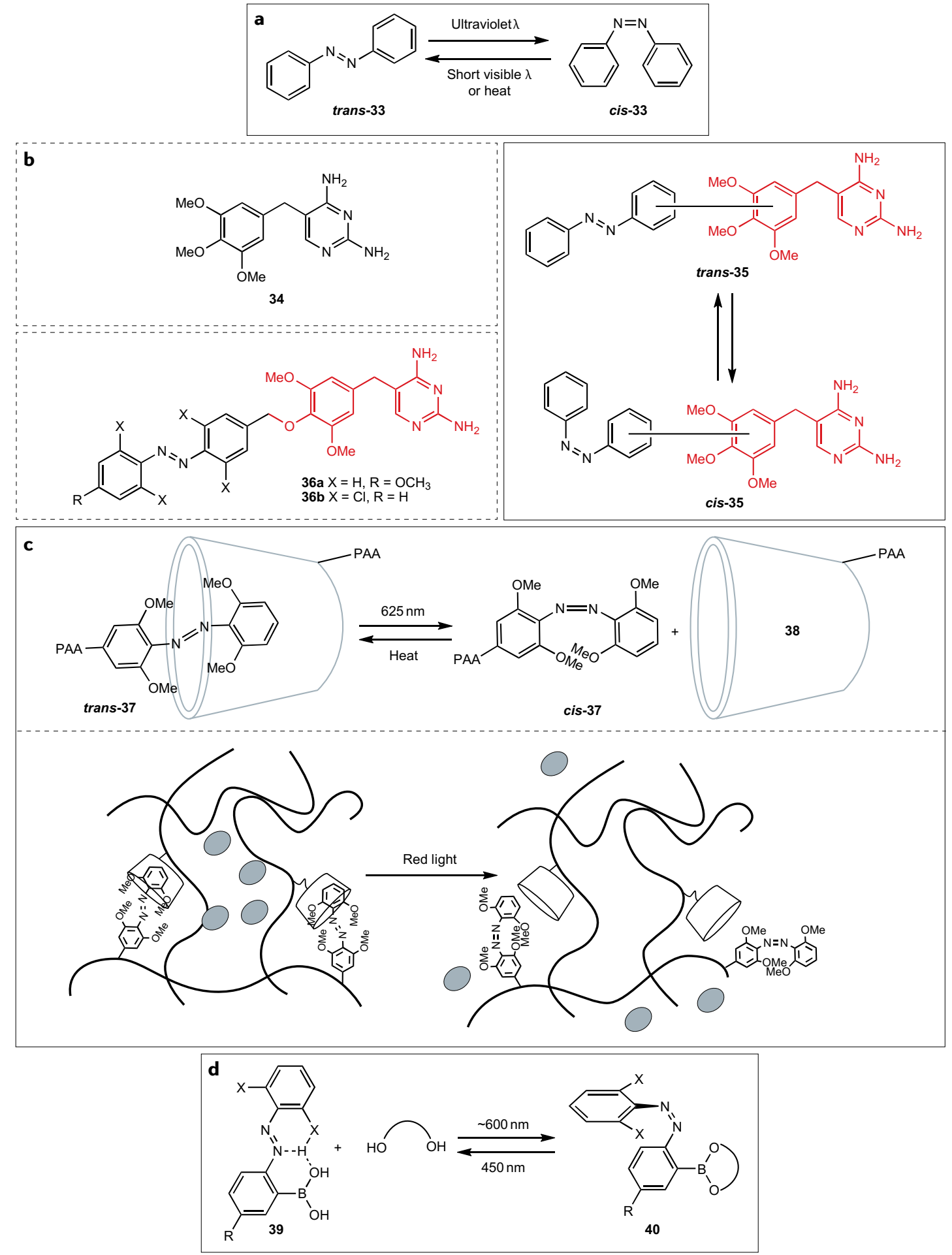

Fig. 6 | Photoisomerization of azobenzene. a | Photoisomerization of azobenzene 33 by ultraviolet and short visible wavelengths. $\mathbf{b} \mid$ The antibiotic trimethoprim $\mathbf{3 4}$ was appended to a library of azobenzene-substituted trimethoprim derivatives (35) that were screened for antibacterial activity. The library was screened for members that displayed a dependence on the cis:trans ratio, which resulted in the identification of the lead derivative $\mathbf{3 6}(\mathrm{X}=\mathrm{Cl}, \mathrm{R}=\mathrm{H})$. c $\mid$ Disruption of the host-guest interaction of $\mathbf{3 7}$ with $\beta$-cyclodextrin $\mathbf{3 8}$ upon 625 -nm-induced trans-to-cis photoconversion. Photorelease $(625 \mathrm{~nm})$ of encapsulated bovine serum albumin (grey circles) from poly(acrylic acid) (PAA)-modified derivatives of an azobenzene (37) and a $\beta$-cyclodextrin hydrogel. $\mathbf{d} \mid$ Photoisomerization of $\mathbf{3 9}$ to $\mathbf{4 0}$ promotes diol binding up to 20 -fold.

on the cis:trans ratio (FIG. 6). Indeed, the library screen furnished a lead (36a; $\left.\mathrm{X}=\mathrm{H}, \mathrm{R}=\mathrm{OCH}_{3}\right)$ in which the $c$ is isomer is a more potent antibiotic than the trans isomer, albeit only by twofold. Although the $\lambda_{\max }$ for $36 \mathrm{a}$ is at $360 \mathrm{~nm}$, irradiation at the tail end of the spectrum (at $537 \mathrm{~nm}$ with $\Phi=0.18$ ) favours the $c i s$ isomer over that 
of its trans counterpart $(\Phi=0.02)$. Red-shifted analogues $\left(\lambda_{\max }=465 \mathrm{~nm}\right.$, but photoresponsive out to $652 \mathrm{~nm}$ ) were constructed via the introduction of halogen substituents $(\mathbf{3 6} \mathbf{b} ; \mathrm{X}=\mathrm{Cl}, \mathrm{R}=\mathrm{H})$, based on previous work $^{121,122}$. However, as noted above, the photoconversion of one azobenzene isomer to the other is rarely absolute. For both 36a and 36b, the optimized photostationary state ratio for cis:trans is $\sim 7: 1$. With the latter in mind, dark state $\mathbf{3 6}$ b (trans isomer favoured) displays a minimum inhibitory concentration to inhibit the growth of $50 \%$ of organisms $\left(\mathrm{MIC}_{50}\right)$ of $>80 \mu \mathrm{M}$. By contrast, the $\mathrm{MIC}_{50}$ for irradiated $36 \mathrm{~b}$ is $10 \mu \mathrm{M}$. The dark:light $\mathrm{MIC}_{50}$ ratio is eightfold and approximates that of the cis:trans ratio of the photostationary state (sevenfold).

The tetra-ortho-methoxy-substituted azobenzene (37) was designed to trigger the release of compounds from hydrogels and mesoporous silica nanoparticles ${ }^{123,124}$.

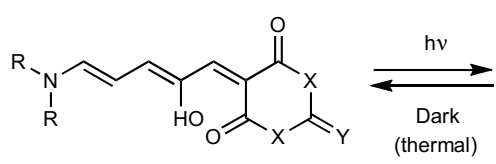

41

b
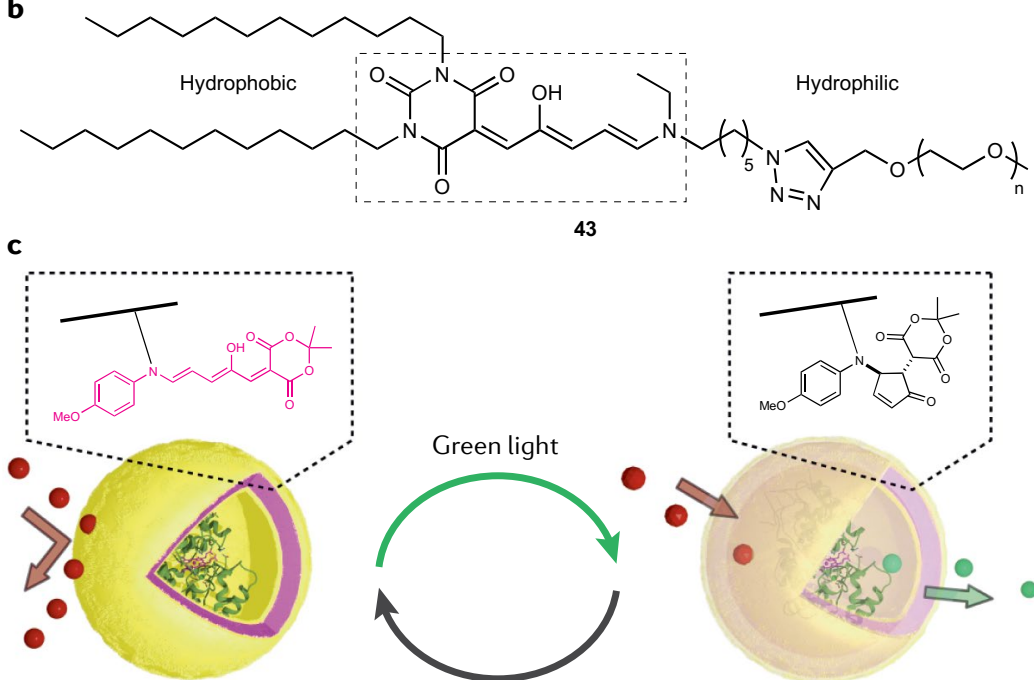

Impermeable shell

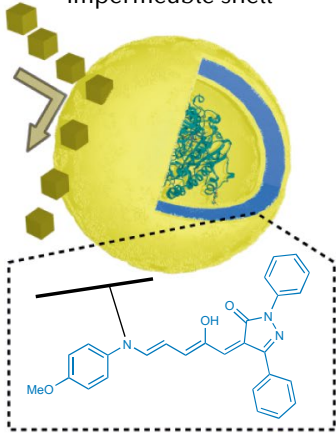

Nanoreactor-off
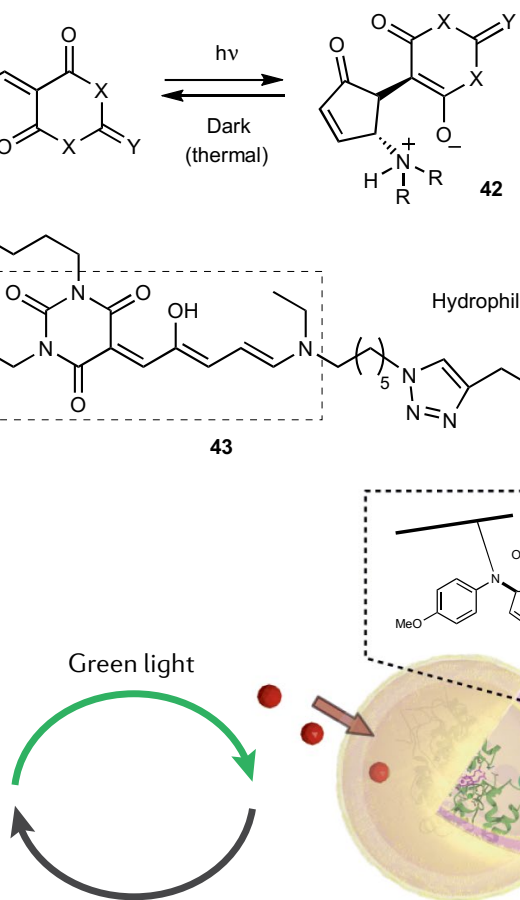

Recovery in dark

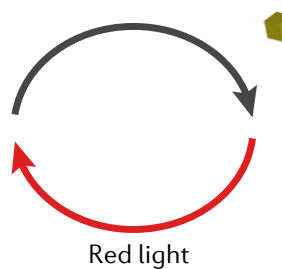

Transient permeability

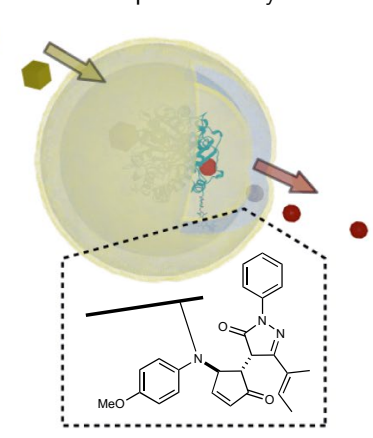

Nanoreactor-on
Fig. 7 | Photoisomerization of donor-acceptor Stenhouse adducts. a | Photoisomerization of donor-acceptor Stenhouse adducts (DASAs) 41 and $\mathbf{4 2 .}$ b| DASA 43 is a zwitterion and is readily accommodated into micelles. $\mathrm{c}$ Polymersomes made of DASA-containing amphiphilic block copolymers are impermeable to small molecules in the dark and permeable upon exposure to the appropriate wavelength of light — upon illumination, micellar integrity is disrupted and can, thereby, release internal cargo. Part c adapted with permission from REF. ${ }^{130}$, American Chemical Society.
The trans azobenzene isomer of $\mathbf{3 7}$ binds to $\beta$-cyclodextrin 20 -fold more tightly than the corresponding cis analogue $^{123,125}$ (FIG. 6). Poly(acrylic acid)-modified derivatives of $\mathbf{3 7}$ and $\beta$-cyclodextrin (38) are mixed to form a hydrogel that is loaded with fluorescent bovine serum albumin (FIG. 6). Subsequent illumination at $625 \mathrm{~nm}$ disrupts the hydrogel and releases more than $80 \%$ of the loaded bovine serum albumin. An analogous strategy has been successfully deployed for doxorubicin, which can be photoreleased from mesoporous silica nanoparticles ${ }^{124}$.

Finally, a boronic-acid-substituted azobenzene has also been used to control the binding of diols ${ }^{126,127}$. In particular, 39 exhibits a greater affinity for various diols (up to 20-fold) than its isomeric counterpart 40. This light-dependent preference for diols has been used to trigger the sol-gel transition of a poly(ethylene glycol) hydrogel.

Scope and opportunities. The azobenzene scaffolds reported to date have modest $\varepsilon$ values in the optical window of tissue. Furthermore, the cis and trans isomers have overlapping wavelengths of absorption. Therefore, excitation generates a photostationary state in which one isomer (typically, the cis) is only moderately favoured over the other. However, these derivatives do offer interesting opportunities as molecular gatekeepers on nanoparticle-based drug delivery systems. Further improvements in the photophysical properties of these derivatives will drive drug delivery efforts.

\section{Donor-acceptor Stenhouse adduct scaffolds}

DASA derivatives have been reported with $\lambda_{\max }$ values of $670 \mathrm{~nm}\left(\mathrm{REF}^{128}{ }^{128}\right.$. These species, as exemplified by $\mathbf{4 1}$, are comprised as an electron-deficient region (Meldrum's or barbituric acids, where $\mathrm{X}=\mathrm{O}$ or $\mathrm{N}$, respectively) and an electron-rich amine-based moiety. Light absorption by $\mathbf{4 1}$ triggers an olefin isomerization and a subsequent thermal electrocyclization, thereby, generating the colourless cyclopentenone derivative $\mathbf{4 2}$ (FIG. 7). Consequently, unlike azobenzenes, illumination-induced isomerization proceeds only in one direction. Reconversion to the triene $\mathbf{4 1}$ transpires in the dark (thermally). In addition, the interconversion of $\mathbf{4 1}$ and $\mathbf{4 2}$ provides a hydrophobic to hydrophilic polarity change, which can be exploited to release therapeutic agents $^{129-132}$.

In one example, the DASA photoswitch was employed to create light-sensitive micelles that house hydrophobic compounds in the dark and release them upon illumination ${ }^{129}$. DASA 41 is an amphiphile that aggregates into micelles. Upon illumination with visible light, photoisomerization of $\mathbf{4 1}$ to its zwitterionic counterpart disrupts the micelle, resulting in the release of cargo (paclitaxel). Unlike the reversible interconversion of $\mathbf{3 9}$ and $\mathbf{4 0}$, micelle disruption is irreversible due to solvation of the zwitterionic isomer ${ }^{129}$.

The light-induced change in hydrophobicity of DASA derivatives has also been used to create light-controlled bioreactors ${ }^{130}$. The latter are of significant biomedical interest, as exemplified by the application of asparaginase for the treatment of acute lymphoblastic leukaemia (a cancer dependent upon a plasma source of 
asparagine $)^{133}$. DASA derivatives were employed to control the membrane permeability of polymersomes, which are hollow polymer vesicles (FIG. 7). Polymersomes, comprised of a DASA-based amphiphilic block copolymer, were rendered permeable to small molecules upon illumination, and they subsequently returned to their impermeable state in the dark. This was demonstrated in several ways, but most relevant to the concept of therapeutically useful bioreactors is the incorporation of glucose oxidase into the polymersome ${ }^{130}$. In the presence of light, glucose enters the polymersome and is acted upon by glucose oxidase, which generates hydrogen peroxide as a by-product. The latter, when coupled with peroxidase, is commonly used to colorimetrically monitor glucose levels.

Scope and opportunities. Unlike the azobenzene derivatives, only one isomer of the DASA scaffold is photoresponsive, with a maximal wavelength of absorption out to $670 \mathrm{~nm}$. In addition, DASA derivatives have been incorporated into carriers. These features are promising, but additional studies are required to improve the photophysical properties of this scaffold and establish therapeutic generality.

\section{Comparison of photoswitchable and photolytic strategies}

The design of phototherapeutics based on the photoswitch mechanism requires identification of a modifiable, non-essential site on the drug of interest. Modification at a non-essential site is necessary, since the photoswitch remains attached to the drug molecule. In addition, the photostationary state of photoswitches is commonly comprised of both isomers, one of which predominates. In short, photoconversion from a completely inactive to active form is challenging. Furthermore, it is not unusual for the less thermodynamically stable isomer to thermally convert to the more stable isomer in the dark. Consequently, it makes sense to engineer bioactivity into the less stable isomer, which can then revert to the inactive form in the dark. Finally, since thermal reversion from one isomer to the other can be fast, this will likely necessitate continuous illumination at the diseased site to ensure the presence of active drug. By contrast, the photolysis mechanism commonly entails modification of a functional group that is essential for activity on the drug of interest. But not all therapeutic agents possess an indispensable or a dispensable functionality that is easily modified. However, despite unique challenges in the design of photoswitchable and photolytic light-activated therapeutics, both strategies do share potential shortcomings. First, covalent modification of a therapeutic agent is less than desirable, since it is the parent structure that has been optimized for therapeutic purposes. Second, approval for the use of modified drugs faces significantly higher hurdles than approval for parent drugs that have simply been reformulated. Third, the vast majority of potential phototherapeutic agents discussed to this point require a photo event for every drug molecule activated. However, the one drug/single photo event constraint is therapeutically inefficient, particularly if large amounts of active drug need to be delivered to the diseased site. Constructs that release or activate multiple drugs with a single photo event represent an attractive alternative, a strategy that necessitates the use of carriers (nanoparticles, hydrogels, cells etc. $)^{3,8,11,123,124,134}$. Indeed, although both photolytic and photoswitchable strategies have been used in this fashion, with few exceptions (FIG. 4 and previous discussions), nearly all examples employ short wavelengths for the photorelease of therapeutically active cargo.

\section{Path to clinical applications}

We have highlighted the current status of the design of long-wavelength-responsive scaffolds and the drugs appended or associated with them. However, like conventional drugs, acquisition of a preclinical lead (for example, a potent inhibitor of a target enzyme) represents just the beginning of an often tortuous path to the final therapeutic agent. For example, the absorption, distribution, metabolism and excretion of a potential drug needs to be addressed ${ }^{135}$. Absorption, distribution, metabolism and excretion, and related issues, may prove to be uniquely challenging, given the structural complexity of phototherapeutics relative to their conventional counterparts. Light-activatable drugs are multicomponent entities that not only include the therapeutic agent to be delivered but also the chromophore, (often) a linker between the chromophore and drug, and, potentially, a carrier. Furthermore, phototherapeutics have the additional issue of dependence on a light source. A 2018 perspective on photoactivated chemotherapy noted that much of the work to date has focused on the acquisition of light-activated drugs with only an obligatory nod to their clinical application ${ }^{43}$. This is perfectly understandable, given the relatively recent introduction of long-wavelength-responsive drug scaffolds. It has also been argued that it is critical to take a disease-based approach in the design of phototherapeutic agents ${ }^{43}$, a sentiment that we share. This diseased-based imperative is addressed in the sections that follow, with a focus on the transport of phototherapeutic agents (in the next section entitled Carriers) and the clinical rationale for the use of light-activated drugs (in the section entitled The phototherapeutic effect).

\section{Carriers}

In earlier sections, we briefly touched on the use of carriers for phototherapeutics. Drug transporters offer a number of potential advantages, relative to that of the phototherapeutic itself, in an in vivo setting. These include the distribution, protection from metabolism and quantal (localized, high-concentration) delivery of the phototherapeutic. In addition, properly designed carriers potentially allow the parent drug, and not a covalently modified analogue, to be conveyed to and photoreleased at the diseased site. The potential beneficial attributes of carriers are outlined below.

Distribution. At first glance, this may appear to be redundant, since one of the presumed strengths of phototherapeutics is light-directed spatial targeting. However, built-in redundancies to accentuate diseased site delivery should be viewed as advantageous. This is exemplified by 
the antibody-phototherapeutic conjugates (19), which preferentially distribute the appended caged drugs to specific sites (including the site of therapeutic interest). Subsequent illumination furnishes enhanced spatially resolved treatment. However, carriers can serve roles beyond active targeting of the diseased sites. For example, stimuli-responsive hydrogels have been investigated as drug depots, including those that respond to $\mathrm{pH}$, temperature and small molecules. Light offers the means to precisely control the timing and amount of drug that is released ${ }^{136}$. Photochemically responsive hydrogelbased drug release systems have also been demonstrated (FIG. 6). A very different strategy uses RBCs to confine therapeutic agents to the vasculature (FIG. 4). Disorders of the vasculature, including ischaemic and haemorrhagic stroke, represent clinical targets of opportunity. It would also be premature to rule out the possibility of using phototherapeutics in the absence of carriers. It is possible that one or more of the chromophorecontaining scaffolds might selectively accrue at diseased sites. For example, in analogy to folate receptor-targeted chemotherapeutics ${ }^{137}, \mathrm{~B}_{12}$-drug conjugates are known to accumulate in tumours ${ }^{138,139}$. However, if the phototherapeutic agent is directly taken up by cells, what happens in the absence of light? Is the drug metabolically cleaved from the chromophore? If not, how long does the phototherapeutic remain functionally silent? Is the phototherapeutic removed from or inactivated in non-illuminated tissue? These are questions that need to be addressed when considering the use of phototherapeutics alone or in conjunction with a carrier.

Metabolism. Carriers possess the potential advantage of sheltering the drug, linker and chromophore from metabolic processing. The metabolic fate of approved drugs is well defined. However, a key challenge associated with the design of peptide and protein-based therapeutics is their metabolic instability, resulting in a limited circulatory lifespan (for example, tissue plasminogen activator, which is on the order of a few minutes) ${ }^{140}$. With this in mind, photorelease of a metabolically sensitive therapeutic, from a carrier, at the diseased site represents a potentially game-changing clinical opportunity. In addition to the drug itself, the linker between drug and chromophore is typically comprised of functionality that is metabolically fragile, such as the carbamate in $\mathbf{4}$, the thiocarbamate in $\mathbf{9}$, the amide in $\mathbf{2 4}$ and $\mathbf{2 5}$, and the ester in $\mathbf{2 6}$. When appropriately embedded within a carrier, the key linker functionality should be protected from circulatory hydrolytic enzymes. Indeed, depending upon the nature of the disease, oral administration may be the preferred route of delivery. Consequently, a carrier, whether artificial (for example, mesoporous silica nanoparticles) or endogenous (for example, transcobalamin), may offer the means by which the phototherapeutic can be orally delivered in an intact form. Finally, in a few instances, chromophore metabolism has been explored. For example, the azobenzenes are known to be prone to reduction by glutathione ${ }^{40}$, an intracellular tripeptide present at concentrations of up to $10 \mathrm{mM}$. Fortunately, electron-rich azobenzenes appear to be more resistant to glutathione-mediated reduction ${ }^{40}$. Alkylcobalamins are, likewise, potentially subject to intracellular metabolic processing, but via reductive dealkylation ${ }^{141}$. In short, carriers may serve to protect the phototherapeutic from metabolic processing in two ways: as a shield against circulatory enzymes and as a vehicle that limits unwanted cellular uptake and/or excretion.

Quantal delivery. Carriers can be loaded with high drug concentrations, which potentially enables delivery of relatively large amounts of therapeutic agent in a localized fashion. This has analogy to the quantal release mechanism of neurotransmitters observed at synapses. Quantal delivery is of paramount importance if high concentrations of a drug are required for therapeutic efficacy, such as if the drug has a modest dosage that is effective in $50 \%$ of the subjects $\left(\mathrm{ED}_{50}\right)$ or a short therapeutic lifespan due to metabolic instability.

Parent drug. We've noted that the design of phototherapeutics has primarily focused on covalently modifying existing drugs with a chromophore. However, a more ideal approach is the use of the unmodified parent drug, such as a small molecule, peptide or protein. Appropriately designed carriers offer this opportunity, which have the potential to deliver a mixture of drugs. To date, there have been two general strategies that exploit the use of parent therapeutic agents; the photogatekeeper approach (FIG. 7), whereby channels for the entry or exit of compounds of interest are controlled by a light-responsive molecular doorway, or, alternatively, the photo-disassembly approach (FIGS 4f,6), which results in the destruction of the carrier and release of internal contents.

In summary, carriers offer several potential advantages with respect to disease application, including the controlled distribution, metabolic protection and quantized delivery of the phototherapeutic agent

\section{The phototherapeutic effect}

The spatially targeted delivery and activation of a drug at a diseased site dominates the clinical rationale for phototherapeutics. Can focused drug delivery to the diseased site reduce the cardiotoxicity associated with doxorubicin, the cerebral haemorrhaging induced by tissue plasminogen activator or the multitude of side effects caused by the long-term use of dexamethasone? In short, the therapeutic promise of light-activated drugs hinges upon the premise of enhanced efficacy and reduced toxicity. This premise can be validated by addressing two key questions associated with the use of light to target disease and the clinical benefits of the released drug in comparison with the parent drug.

Question 1. Can light be used to activate or release drugs at targeted diseased sites? Recently acquired data support the notion that spatial targeting is an established property of phototherapeutics. For example, the light-triggered NO generator 6 can be site-specifically activated in vivo in a fashion that supports tumour suppressive activity ${ }^{55}$. A key design element of this study was the implantation of two tumours (one in each flank) of the mouse model, thereby, providing an internal control (one tumour illuminated and the other kept in the dark). 
Acoustic angiography An imaging technique that uses ultrasound in combination with microbubbles as a contrast agent.
Irradiation furnished confirmation of NO release via visualization of the BODIPY by-product (7), which was not observed in the non-illuminated tumour (FIC. 8). Second, several important control experiments were performed to establish that both light $(700 \mathrm{~nm})$ and $\mathbf{6}$ are necessary for the desired therapeutic outcome: (1) vehicle control (that is, injection without phototherapeutic) in the absence of light, (2) vehicle control with light, (3) injected phototherapeutic without light and (4) injected phototherapeutic with light. These treatment regimens were repeated every other day over the course of a week. The tumour size in the experimental group (phototherapeutic + light) was half that of the control groups. Phototherapeutic 6 was not only taken up by the tumour but was found in the heart, liver, kidneys and spleen as well. This is not surprising, given the untargeted nature of the phototherapeutic. However, the animals did not suffer from any general signs of toxicity (weight loss or behavioural changes), indicating that, if 6 is metabolized in non-illuminated tissue, the effect is non-toxic.

The antibody-phototherapeutic conjugate 19 was, likewise, investigated for its antitumour properties, but, in this case, the potent cytotoxin duocarmycin was photochemically released ${ }^{67}$. The phototherapeutic was introduced by tail vein injection in tumour-bearing mice. As expected, the antibody-phototherapeutic accumulated at the tumour, but it was also present in the bladder and liver. Photoactivation $(780 \mathrm{~nm})$ at the tumour site resulted in a decrease in tumour volume, as well as an increase in survival using moderate and high doses of phototherapeutic (FIG. 9). Controls included vehicle only, as well as treatment with phototherapeutic 19, but no illumination. Interestingly, for the latter
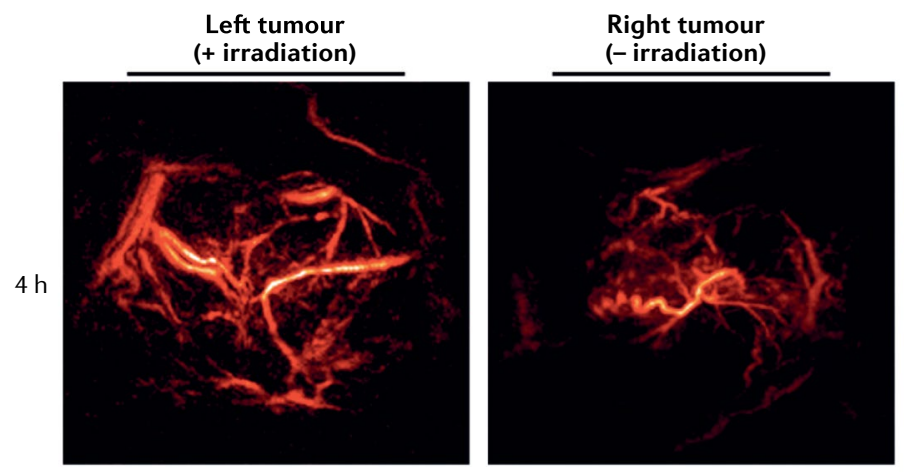

$\operatorname{Max}$
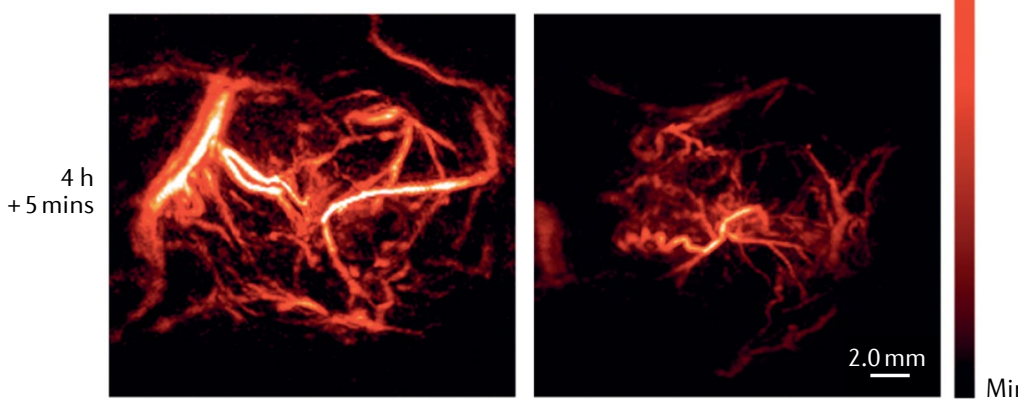

Fig. 8 | In vivo photogeneration of NO. Photoacoustic tomography image of the NO generator 6 (FIG. 2) in the left and right tumours of a mouse model acquired before (top) and after (bottom) 5 min irradiation (left) or dark control (right). The enhanced photoacoustic signal in the irradiated left tumour (bottom left) is due to the formation of the by-product 8 (FIG. 2). Adapted with permission from REF. ${ }^{55}$, American Chemical Society. non-irradiated control, a modest therapeutic effect was observed. It is unclear whether this was due to inadvertent activation of 19 by ambient room light or through a pathway involving antibody-drug tumour uptake and metabolic drug release from the antibody.

Cobalamin scaffolds have also been used to deliver therapeutic agents in a targeted fashion in vivo ${ }^{79}$. RBCs containing the phototherapeutic $\mathbf{4 4}$ were tail vein injected into tumour-bearing mice and the tumour subsequently illuminated at $655 \mathrm{~nm}$. Unlike the studies with the NO generator 6 and the antibody-conveyed duocarmycin 19, the in vivo studies with the taxol derivative 44 did not address the questions of tumour burden or animal survival. Rather, the experiments explored the immediate impact $(<1 \mathrm{~h})$ of taxol release on tumour blood vessel integrity. Acoustic angiography revealed uptake of contrast reagent by the tumour, consistent with the notion that taxol compromised blood vessel integrity within the immediate vicinity of the tumour. Controls included vehicle only (that is, unloaded RBCs), treatment with phototherapeutic but no illumination and illumination of a non-therapeutic (45). The latter contains the Cy5 antenna, which can potentially act as a photodynamic agent, but is lacking the taxol derivative. Only 44 with illumination compromised tumour blood vessel integrity. A Cbl scaffold has also been used to trigger the photohaemolysis of RBCs and, thereby, release protein therapeutics ${ }^{85}$ (FIG. 4f). In this case, a blood clot was site-specifically triggered in the ear of mice via the release of thrombin (FIG. 10). Once again, dark/light, as well as empty RBCs, were used as controls to validate the absolute requirement for RBCs, the phototherapeutic and light.

In summary, experiments with long-wavelengthresponsive phototherapeutics have demonstrated the light-triggered, in vivo site-targeted release of therapeutic agents, including gas molecules ( $\mathrm{NO}$ and $\mathrm{CO}$ ), small molecule cytotoxins and a protein. All of these studies incorporated key control experiments establishing that both the phototherapeutic and light are necessary to observe the anticipated biological impact.

Question 2. Does light-targeted drug therapy provide a therapeutic benefit relative to treatment with the parent drug? This is a critically important question with respect to obtaining approval for clinical use. However, the inherent bias of this question ignores a potentially transformative attribute of phototherapeutics: the ability to salvage bioactive species that are simply too toxic for use as systemically delivered therapeutic agents. A few examples are highlighted in the previous section: $\mathrm{NO}$ (and CO), the incredibly cytotoxic duocarmycins ${ }^{142}$ and thrombin. Indeed, the latter is a mediator of the extreme increased blood clotting observed in some COVID-19 patients $^{143}$. In short, phototherapeutics not only offer the means to improve the therapeutic outcome of existing drugs but also provide an opportunity to transform profoundly malicious or insufficiently effective agents into pharmacologically acceptable drugs. In this regard, phototherapeutics are the prospective descendants of Ehrlich's magic bullet ideal, which serves as the conceptual framework for creating therapeutic agents with exemplary safety profiles. However, 


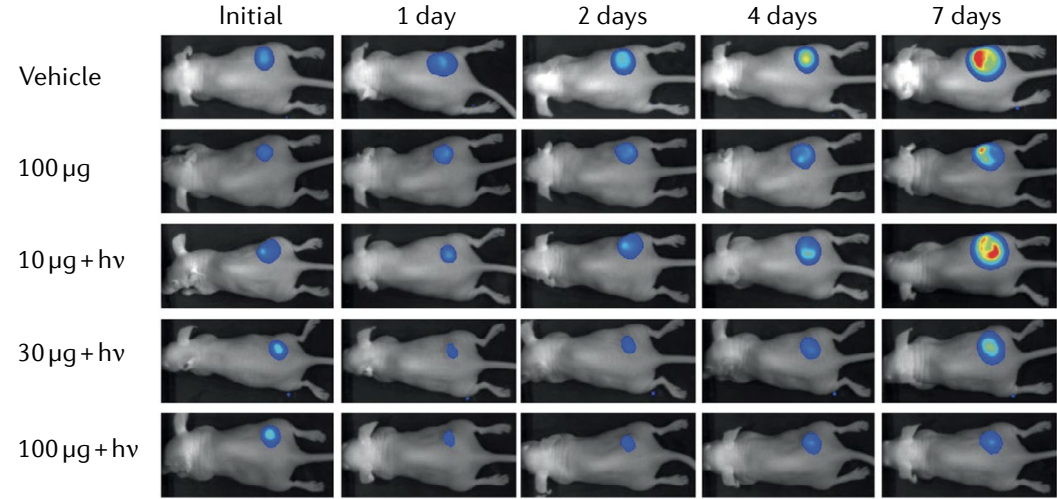

Fig. 9 | In vivo photorelease of cyanine-caged cargo. Bioluminescence imaging of a luciferase-containing tumour in mice receiving the following treatments: vehicle control (first row), $100 \mu \mathrm{g}$ antibody-phototherapeutic 19/no light (second row), $10 \mu \mathrm{g}$ antibodyphototherapeutic 19/light (third row), $30 \mu \mathrm{g}$ antibody-phototherapeutic 19/light (fourth row), $100 \mu \mathrm{g}$ antibody-phototherapeutic 19/light (fifth row). The 30- $\mu \mathrm{g}$ and $100-\mu \mathrm{g}$ treatments furnished a reduced tumour burden at day 7 relative to the vehicle control. See the narrative for a brief discussion of the beneficial effect observed with the phototherapeutic in the absence of light (second row). Adapted with permission from REF. ${ }^{67}$, American Chemical Society. demonstrating the ability to spatially deliver and/or temporally control the action of a phototherapeutic is only a first step. These experimental results, no matter how impressive, will not sway the 'key opinion leaders" ${ }^{43}$ that phototherapeutics represent a clinically viable technology. What will be needed? First, it is critical that phototherapeutics are applied to the right disease ${ }^{43}$. A few examples are discussed in BOX 1 . Second, key preclinical quantitative measures of efficacy, in the context of safety, are essential. However, these quantitative assessments do not exist in a vacuum. A comparison with the standard of care, with an emphasis on parent drugs, is a prerequisite in establishing phototherapeutics as a clinically viable technology. What do these quantitative comparative assessments entail?

The therapeutic index (TI) is a key parameter that is an essential component of drug development ${ }^{144}$. The TI is furnished as a ratio; for example, the drug dose that induces toxicity in $50 \%$ of the subjects $\left(\mathrm{TD}_{50}\right)$ divided by the drug dose that is effective in $50 \%$ of the subjects $\left(\mathrm{ED}_{50}\right)$. A drug with a large TI has a good safety profile, indicating a small likelihood of an adverse response. However, with phototherapeutics, there are two forms of the drug: the dark (presumably inactive) precursor and the light-activated species. A ratio somewhat analogous to the TI that measures the phototoxicity of drugs is referred to as the phototoxic index. For example, this may take the form of the cytotoxicity (or, in general, bioactivity) of a drug in the dark versus that in the light $\left(\mathrm{IC}_{50 \text { (dark) }} / \mathrm{IC}_{50 \text { (light) }}\right)$. A large phototoxic index is consistent with a strong light dependency for bioactivity ${ }^{145}$. Given the possibility that ambient light or metabolism may activate the phototherapeutic, it is not unreasonable to assume that a pharmacologically beneficial effect may be observed in the absence of illumination (FIG. 9). We believe that it is critical to consider the potential pharmacological and toxicological effects of both the 'dark/inactive' and 'light/active' forms of the phototherapeutic, since both are present during the course of the therapy.

In addition to controlling when and where a drug is activated, light can be used to control the dosing regimen of the drug at the diseased site (for example, a single photo-bolus release versus multiple light pulses over a given period of time). Indeed, the dosing regimen is a potentially critical parameter that could affect the safety profile. For example, photorelease of a rapidly metabolized therapeutic agent from a carrier could have a more beneficial effect if it is intermittently released over time versus all at once. In addition to the dosing regimen, we've alluded to the possibility of metabolic activation or deactivation of phototherapeutics. Light-activated drugs that are rapidly metabolized in the dark provide only a small window for phototherapy, whereas more metabolically robust agents offer longer term options. A detailed pharmacokinetics analysis will furnish insight into the time frame of the phototherapeutic window. Finally, a phototherapeutic designed to replace a conventional drug will not only need to demonstrate enhanced efficacy but also a mechanism of action that recapitulates the parent drug following photoactivation. Consequently, a detailed pharmacodynamics study is warranted as well.

\section{Conclusions}

The acquisition of long-wavelength fluorophores and an array of imaging modalities have been combined to detect diseased targets deep within tissue $\mathrm{e}^{146}$. However, light has been primarily limited for viewing (as opposed to modulating) in vivo biology and biochemistry, with PDT and optogenetics serving as notable exceptions. With the advent of long-wavelength-responsive drug delivery systems, in combination with breakthroughs in light delivery technology, it is now possible to use photons to perform well-defined actions at the tissue, cellular and biochemical levels. Nonetheless, although promising, there are challenges that this emerging technology must address if clinical applications are to be realized:

1. Most photoresponsive scaffolds developed to date have been directly attached to drugs. However, not all drugs have readily accessible attachment sites. In addition, covalent modification of protein-based therapeutics is non-trivial, particularly from a manufacturing perspective. We suspect that many (if not all) of the long-wavelength scaffolds described in this Review can also find utility as light-responsive gatekeepers on drug carriers.

2. Attention should be given to the creation of phototherapeutics that can be delivered via oral, sublingual or ocular routes. Although intravenous, intramuscular and subcutaneous injections are certainly appropriate for medical emergencies and other acute conditions, we envision that chronic diseases, involving patient self-treatment, are best supported by more convenient delivery routes.

3. Additional light-absorbing scaffolds in the optical window of tissue should be developed, with an emphasis on the acquisition of a palate of scaffolds that can be wavelength-differentiated. 

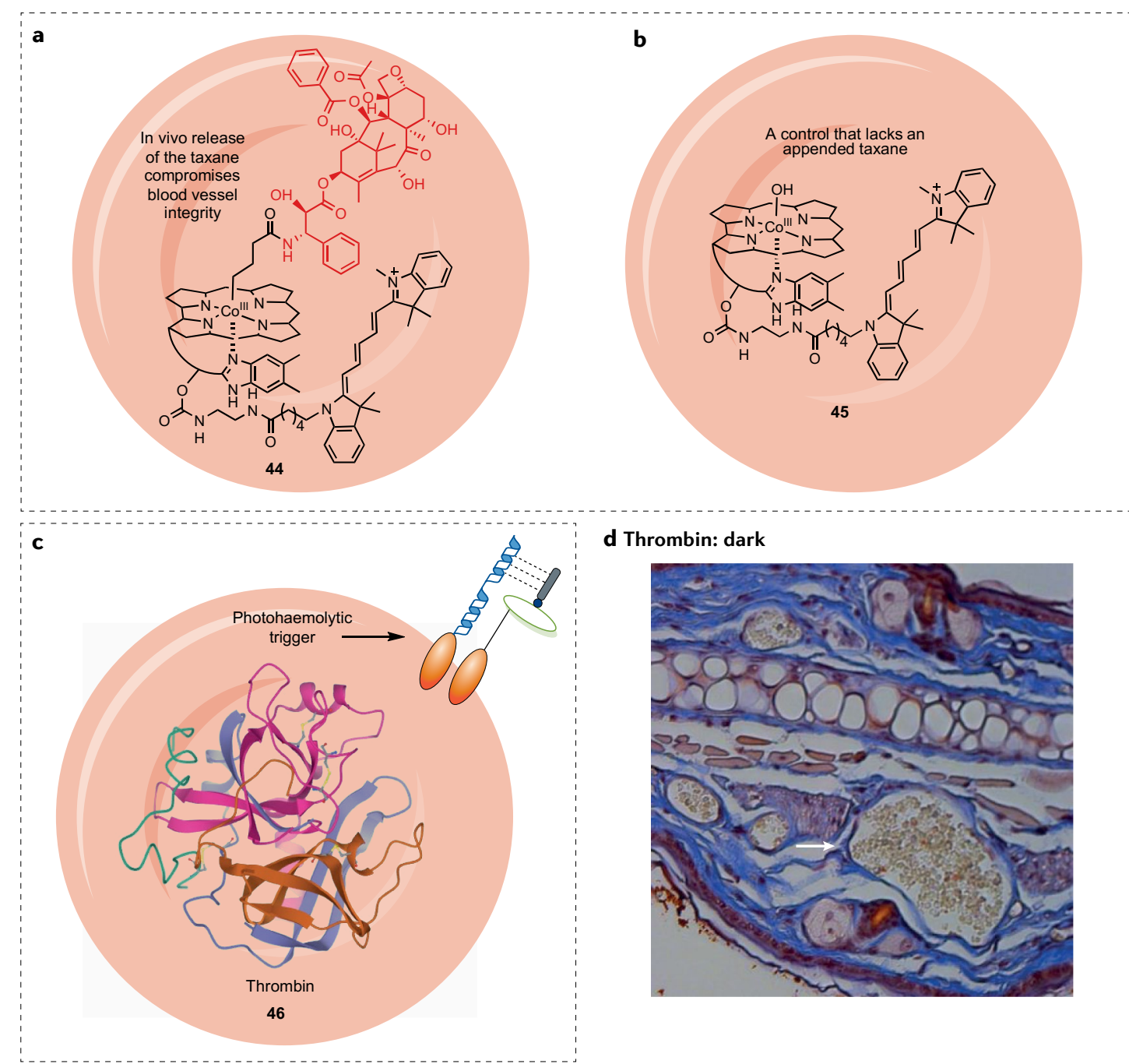

d Thrombin: dark

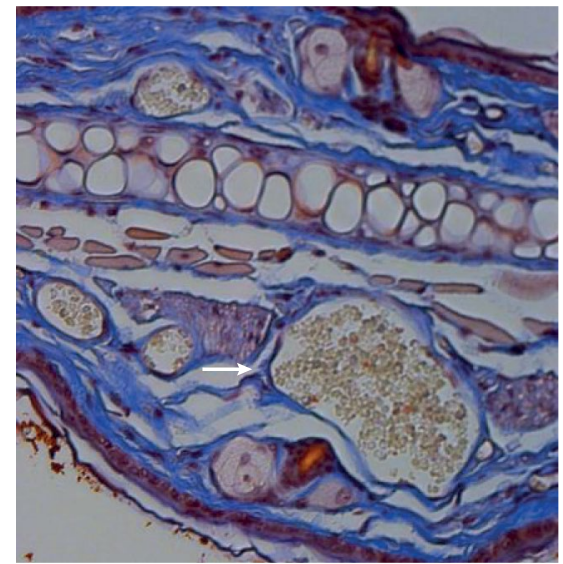

e Mock: buffer light

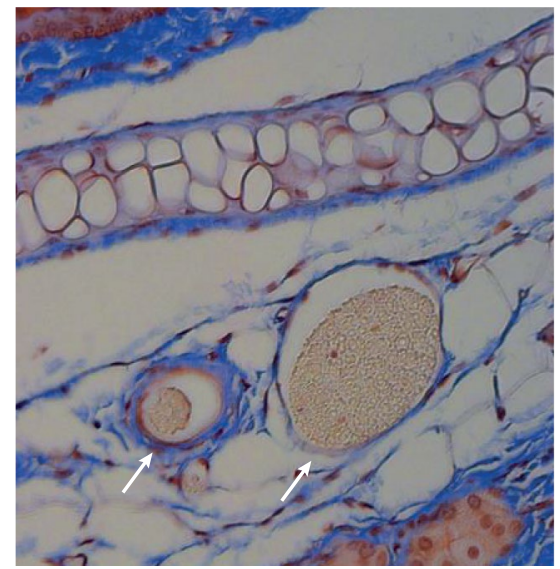

f Thrombin: light

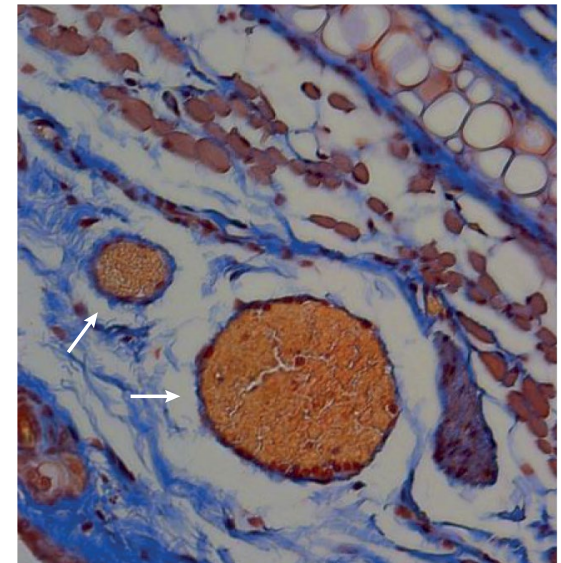

Fig. 10 | Photohaemolytic trigger in RBCs. a | Depiction of a red blood cell (RBC) bearing a taxane-cobalamin conjugate (44), which results in the loss of blood vessel integrity ${ }^{79}$. $\mathbf{b} \mid$ Analogous experiments with RBCs containing a control (45) that lacks the taxane have no impact on the vasculature. $c \mid$ RBCs containing the blood clotting enzyme thrombin (PDB-2HNT) (46) and a membrane-appended photohaemolytic trigger (FIG. 4d-f). d-f $\mid$ Following treatment, mice were sacrificed and tissue was stained and sectioned (RBCs in yellow, collagen in blue and fibrin in orange/red). Blood vessels contain collagen (blue stain) and are highlighted with white arrows. $\mathbf{d}$ | Treatment with $\mathbf{4 6}$ but tissue was not irradiated. RBCs are observed with empty space within the blood vessel, consistent with a healthy vasculature. $\mathbf{e} \mid$ Analogous observations are seen with mice treated with RBCs containing buffer and treated with light. $\mathbf{f}$ | Illuminated tissue from a mouse injected with $\mathbf{4 6}$ displays the consequences of thrombin release: formation of the blood clotting protein fibrin (orange/red) and venous congestion (absence of empty space between RBCs and the vessel wall), consistent with the presence of a blood clot. Parts $\mathbf{d}-\mathbf{f}$ adapted with permission from REF. ${ }^{85}$, American Chemical Society. 


\section{Box 1 | Prospective applications of phototherapeutics}

The ability to spatially target diseased sites with temporally controlled delivery of one or more therapeutic agents are attributes unique to phototherapeutics. Directed precision dosing has ramifications in nearly all areas of biomedicine, including acute, chronic, local and systemic diseases. A few examples of the biomedical applications of phototherapeutics are considered here, some of which are purposely intended to be provocative.

Metastatic cancer: phototherapeutics have long been advocated for use as cancer chemotherapeutics ${ }^{15,43}$. However, this objective has clinical relevance only if it improves upon the current standard of care. Cancers that have the most compelling need for a technological paradigm shift are metastatic and genetically heterogeneous ${ }^{148-150}$. Unfortunately, the absence of one or a few mutational drivers in these diseases advocates for the use of extremely toxic drug cocktails. The potential advantage of phototherapeutics is a greater safety margin than their conventional counterparts. Second, if feasible, most malignant solid tumours are removed by surgery. However, metastatic spread from the surgical site is a recognized phenomenon ${ }^{151}$. Light-triggered drug activation at the tumour offers the ability to wage a vigorous therapeutic presurgery and/or post-surgery campaign, with the goal of reducing or eliminating disease re-emergence. Third, a therapeutic assault on a tumour liberates tumour-associated antigens, which can potentially activate and locally educate $C D 8^{+} T$ cells to attack the parent tumour and metastatic lesions ${ }^{152,153}$. Unfortunately, the immunosuppressive tumour microenvironment interferes with $T$ cell activation. Consequently, there is intense interest in developing strategies for the targeted delivery of immunomodulators to the tumour site ${ }^{153-155}$.

Rheumatoid arthritis (RA): RA is a chronic disease and, therefore, therapeutic administration is frequent and long term, which results in moderate to severe side effects ${ }^{156}$. It has been argued that "successful new therapies [for RA] need not only be efficacious with a good or improved safety profile but must also be formulated to allow for self-directed administration by patients" ${ }^{157}$. Phototherapeutics could allow patients to control when and where the drug is delivered by using hand-held light sources ${ }^{158}$.

Microbial infections: it is estimated that nearly 36,000 Americans die each year from drug-resistant microbes ${ }^{159,160}$. Although the major cause of death is from systemic disease (multisite or whole body), treating localized infections with systemically delivered drugs has exacerbated the problem of drug resistance. Spatially localized infections include those found in surgical wounds, burns, diabetic ulcers, soft tissue (for example, the notorious necrotizing fasciitis or 'flesh-eating disease'), the urinary tract, periodontal tissue, the gastric mucosa (for example, peptic ulcer disease and gastric cancer) and the nasal passage (sinusitis) ${ }^{161}$. A vigorous photoantimicrobial intervention on a spatially localized infection could reduce serious side effects and prevent the disease from becoming systemic.

Disorders of the vasculature: stroke is second only to heart disease as the leading cause of death in the world ${ }^{162}$. Nearly $90 \%$ of all strokes are due to the formation of a blood clot in the brain, and the only pharmacological therapeutic approved as a thrombolytic agent for stroke is a recombinant version of tissue plasminogen activator. Unfortunately, there are serious side effects, which include life-threatening cerebral haemorrhages and life-altering neurotoxicity. Consequently, there is intense interest in identifying technologies that activate tissue plasminogen activator only at the thrombus site ${ }^{163,164}$.

4. Long-wavelength-responsive phototherapeutics with large $\varepsilon \Phi$ values are potentially both a blessing and a curse. It is unclear what impact, if any, ambient light may have on the inadvertent activation of these agents. Consequently, it is critical to obtain a quantitative assessment of background drug release/ activation as a function of ambient light intensity (ranging from the equivalent of bright sunlight to a dimly lit room).

5. The pharmacokinetics of phototherapeutics will be required to address metabolic stability. Metabolic processing could render the phototherapeutic inactive (for example, non-responsive to illumination) and/or transformed to the active drug.

6. New long-wavelength-activated scaffolds should be thoroughly examined for toxicity. Fortunately, these scaffolds employ fluorophores (and closely related derivatives) that have been extensively studied in animal models. Indeed, indocyanine green, a dye structurally related to Cy5, is approved for human use.

7. Although we have emphasized the potential clinical advantages of long-wavelength-responsive phototherapeutics, it is important to acknowledge that short-wavelength-activated drugs may be of therapeutic utility as well. Perhaps the most obvious application is where the diseased site is easily accessible (for example, the skin) and/or where deep tissue penetration is not necessary or potentially harmful. In short, to reiterate a point made earlier, "it is critical to take a disease-based approach in the design of phototherapeutic agents" ${ }^{43}$.

More than a century has passed since Paul Ehrlich advocated for learning how to aim chemically at diseased sites. Ehrlich died in 1915, a decade before the discovery of penicillin, three decades before streptomycin was given to the first patient with tuberculosis, seven decades before the approval of ivermectin for the treatment of river blindness and nine decades before the first protein kinase inhibitor found its way into the clinic. In each of these cases, the clinical experience is characterized by side effects due to undesired activity at otherwise healthy tissue, a common and potentially life-threatening concern with many drugs. Phototherapeutics represents a novel variant on Ehrlich's vision for aiming drugs at diseased sites, but with photons serving as the magic bullets to illuminate the path for therapeutic agents.

\section{Published online 6 October 2021}

\footnotetext{
Williams, K. J. The introduction of 'chemotherapy' using arsphenamine-the first magic bullet. J. $R$. Soc. Med. 102, 343-348 (2009).

2. Strebhardt, K. $\&$ Ullrich, A. Paul Ehrlich's magic bullet concept: 100 years of progress. Nat. Rev. Cancer $\mathbf{8}$, 473-480 (2008)

3. Timko, B. P., Dvir, T. \& Kohane, D. S. Remotely triggerable drug delivery systems. Adv. Mater. 22 4925-4943 (2010).

4. Rahoui, N., Jiang, B, Taloub, N. \& Huang, Y, D. Spatio-temporal control strategy of drug delivery systems based nano structures. J. Control. Release 255, 176-201 (2017).

5. Wells, C. M. et al. Stimuli-responsive drug release from smart polymers. J. Funct. Biomater. 10, 34 (2019).

6. Liu, G., Lovell, J. F., Zhang, L. \& Zhang, Y. Stimulus-responsive nanomedicines for disease diagnosis and treatment. Int. J. Mol. Sci. 21, 6380 (2020).
}

7. Zhou, Y., Ye, H., Chen, Y., Zhu, R. \& Yin, L. Photoresponsive drug/gene delivery systems. Biomacromolecules 19, 1840-1857 (2018)

8. Rapp, T. L. \& DeForest, C. A. Visible light-responsive dynamic biomaterials: going deeper and triggering more. Adv. Healthc. Mater. 9, e1901553 (2020).

9. Shim, G. et al. Light-switchable systems for remotely controlled drug delivery. J. Control. Release 267 , 67-79 (2017).

10. Yang, Y., Mu, J \& Xing, B. Photoactivated drug delivery and bioimaging. Wiley Interdiscip. Rev. Nanomed. Nanobiotechnol. 9, e1408 (2017)

11. Fomina, N., Sankaranarayanan, J. \& Almutairi, A Photochemical mechanisms of light-triggered release from nanocarriers. Adv. Drug Deliv. Rev. 64 1005-1020 (2012)

12. Kwiatkowski, S. et al. Photodynamic therapymechanisms, photosensitizers and combinations. Biomed. Pharmacother. 106, 1098-1107 (2018).
13. Rkein, A. M. \& Ozog, D. M. Photodynamic therapy Dermatol. Clin. 32, 415-425 (2014)

14. Chilakamarthi, U. $\&$ Giribabu, L. Photodynamic therapy: past, present and future. Chem. Rec. 17 775-802 (2017).

15. Reessing, F. \& Szymanski, W. Beyond photodynamic therapy: light-activated cancer chemotherapy. Curr. Med. Chem. 24, 4905-4950 (2017).

16. Hamblin, M. R. Photodynamic therapy for cancer: what's past is prologue. Photochem. Photobiol. 96, 506-516 (2020).

17. Mallidi, S. et al. Beyond the barriers of light penetration: strategies, perspectives and possibilities for photodynamic therapy. Theranostics 6, 2458-2487 (2016).

18. O'Banion, C. P. \& Lawrence, D. S. Optogenetics: a primer for chemists. Chembiochem 19, 1201-1216 (2018).

19. Nazempour, R., Zhang, Q., Fu, R. \& Sheng, X. Biocompatible and implantable optical fibers and 
waveguides for biomedicine. Materials 11, 1283 (2018).

20. Humar, M. et al. Toward biomaterial-based implantable photonic devices. Nanophotonics 6 414-434 (2017)

21. Kim, A. et al. An implantable ultrasonically-powered micro-light-source ( $\mu$ Light) for photodynamic therapy. Sci. Rep. 9, 1395 (2019).

22. Palao, E. et al. Transition-metal-free CO-releasing BODIPY derivatives activatable by visible to NIR light as promising bioactive molecules. J. Am. Chem. Soc. 138, 126-133 (2016)

23. Mitra, K., Lyons, C. E. \& Hartman, M. C. T. A platinum(II) complex of heptamethine cyanine for photoenhanced cytotoxicity and cellular imaging in near-IR light. Angew. Chem. Int. Ed. 57, 10263-10267 (2018)

24. Umeda, N. et al. Boron dipyrromethene as a fluorescent caging group for single-photon uncaging with long-wavelength visible light. ACS Chem. Biol. 9 2242-2246 (2014).

25. Peterson, J. A. et al. Family of BODIPY photocages cleaved by single photons of visible/near-infrared light. J. Am. Chem. Soc. 140, 7343-7346 (2018).

26. Rodgers, Z. L. et al. $B_{12}$-mediated, long wavelength photo-polymerization of hydrogels. J. Am. Chem. Soc. 137, 3372-3378 (2015).

27. Aujard, I. et al. o-Nitrobenzyl photolabile protecting groups with red-shifted absorption: syntheses and uncaging cross-sections for one- and two-photon excitation. Chem. Eur J. 12, 6865-6879 (2006).

28. Gug, S. et al. Photolabile glutamate protecting group with high one- and two-photon uncaging efficiencies. Chembiochem 9, 1303-1307 (2008).

29. Chen, G., Qiu, H., Prasad, P. N. \& Chen, X. Upconversion nanoparticles: design, nanochemistry, and applications in theranostics. Chem. Rev. 114 5161-5214 (2014)

30. Bort, G., Gallavardin, T., Ogden, D. \& Dalko, P. I. From one-photon to two-photon probes: "caged" compounds, actuators, and photoswitches. Angew. Chem. Int. Ed. 52, 4526-4537 (2013).

31. Strangman, G. E., Zhang, Q. \& Li, Z. Scalp and skull influence on near infrared photon propagation in the Colin 27 brain template. Neuroimage 85, 136-149 (2014).

32. Haeussinger, F. B. et al. Simulation of near-infrared light absorption considering individual head and prefrontal cortex anatomy: implications for optical neuroimaging. PLOS ONE 6, e26377 (2011).

33. Okada, E. \& Delpy, D. T. Near-infrared light propagation in an adult head model. I. Modeling of low-level scattering in the cerebrospinal fluid layer. Appl. Opt. 42, 2906-2914 (2003).

34. Jagdeo, J. R., Adams, L. E., Brody, N. I. \& Siegel, D. M Transcranial red and near infrared light transmission in a cadaveric model. PLOS ONE 7, e47460 (2012).

35. Tedford, C. E., DeLapp, S., Jacques, S. \& Anders, J. Quantitative analysis of transcranial and intraparenchymal light penetration in human cadaver brain tissue. Lasers Surg. Med. 47, 312-322 (2015).

36. Lapchak, P. A. et al. Transcranial near-infrared laser transmission (NILT) profiles $(800 \mathrm{~nm})$ : systematic comparison in four common research species. PLOS ONE 10, e0127580 (2015)

37. Pitzschke, A. et al. Red and NIR light dosimetry in the human deep brain. Phys. Med. Biol. 60, 2921-2937 (2015).

38. Henderson, T. A. \& Morries, L. D. Near-infrared photonic energy penetration: can infrared phototherapy effectively reach the human brain? Neuropsychiatr. Dis. Treat. 11, 2191-2208 (2015).

39. Klan, P. et al. Photoremovable protecting groups in chemistry and biology: reaction mechanisms and efficacy. Chem. Rev. 113, 119-191 (2013).

40. Beharry, A. A. \& Woolley, G. A. Azobenzene photoswitches for biomolecules. Chem. Soc. Rev. 40, 4422-4437 (2011)

41. Hull, K., Morstein, J. \& Trauner, D. In vivo photopharmacology. Chem. Rev. 118, 10710-10747 (2018).

42. Silva, J. M., Silva, E. \& Reis, R. L. Light-triggered release of photocaged therapeutics - Where are we now? J. Control. Release 298, 154-176 (2019).

43. Bonnet, S. Why develop photoactivated chemotherapy? Dalton Trans. 47, 10330-10343 (2018)

44. Ellis-Davies, G. C. Caged compounds: photorelease technology for control of cellular chemistry and physiology. Nat. Methods 4, 619-628 (2007).

45. Brieke, C., Rohrbach, F., Gottschalk, A., Mayer, G. \& Heckel, A. Light-controlled tools. Angew. Chem. Int. Ed. 51, 8446-8476 (2012)
46. Ankenbruck, N., Courtney, T., Naro, Y. \& Deiters, A Optochemical control of biological processes in cells and animals. Angew. Chem. Int. Ed. 57, 2768-2798 (2018).

47. Dcona, M. M., Mitra, K. \& Hartman, M. C. T. Photocontrolled activation of small molecule cancer therapeutics. RSC Med. Chem. 11, 982-1002 (2020).

48. Weinstain, R., Slanina, T., Kand, D. \& Klan, P. Visibleto-NIR-light activated release: from small molecules to nanomaterials. Chem. Rev. 120, 13135-13272 (2020).

49. Sitkowska, K. et al. Red-light-sensitive BODIPY photoprotecting groups for amines and their biological application in controlling heart rhythm. Chem. Commun 56, 5480-5483 (2020)

50. Slanina, T. et al. In search of the perfect photocage: structure-reactivity relationships in meso-methyl BODIPY photoremovable protecting groups. J. Am. Chem. Soc. 139, 15168-15175 (2017).

51. Sitkowska, K., Feringa, B. L. \& Szymanski, W. Greenlight-sensitive BODIPY photoprotecting groups for amines. J. Org. Chem 83, 1819-1827 (2018).

52. Goswami, P. P. et al. BODIPY-derived photoremovable protecting groups unmasked with green light. J. Am Chem. Soc. 137, 3783-3786 (2015).

53. Rubinstein, N., Liu, P., Miller, E. W. \& Weinstain, R. meso-Methylhydroxy BODIPY: a scaffold for photolabile protecting groups. Chem. Commun. $\mathbf{5 1}$, 6369-6372 (2015).

54. Shrestha, P. et al. Efficient far-red/near-IR absorbing BODIPY photocages by blocking unproductive conical intersections. J. Am. Chem. Soc. 142, 15505-15512 (2020).

55. Zhou, E. Y. et al. Near-infrared photoactivatable nitric oxide donors with integrated photoacoustic monitoring. J. Am. Chem. Soc. 140, 11686-11697 (2018).

56. Fraix, A., Marino, N. \& Sortino, S. Phototherapeutic release of nitric oxide with engineered nanoconstructs. Top. Curr. Chem. 370, 225-257 (2016).

57. Stacko, P., Muchova, L., Vitek, L. \& Klan, P. Visible to NIR light photoactivation of hydrogen sulfide for biological targeting. Org. Lett. 20, 4907-4911 (2018).

58. Lefer, D. J. A new gaseous signaling molecule emerges: cardioprotective role of hydrogen sulfide. Proc. Natl Acad. Sci. USA 104, 17907-17908 (2007).

59. Beltowski, J., Wojcicka, G. \& Jamroz-Wisniewska, A. Hydrogen sulfide in the regulation of insulin secretion and insulin sensitivity: implications for the pathogenesis and treatment of diabetes mellitus. Biochem. Pharmacol. 149, 60-76 (2018).

60. Mani, S., Untereiner, A., Wu, L. \& Wang, R. Hydrogen sulfide and the pathogenesis of atherosclerosis. Antioxid. Redox Signal. 20, 805-817 (2014).

61. Paul, B. D. \& Snyder, S. H. Gasotransmitter hydrogen sulfide signaling in neuronal health and disease. Biochem. Pharmacol. 149, 101-109 (2018).

62. Pinto, M. N. \& Mascharak, P. K. Light-assisted and remote delivery of carbon monoxide to malignant cells and tissues: photochemotherapy in the spotlight. J. Photochem. Photobiol. C 42, 100341 (2020).

63. Boczkowski, J., Poderoso, J. J. \& Motterlini, R. $\mathrm{CO}-$ metal interaction: vital signaling from a letha gas. Trends Biochem. Sci. 31, 614-621 (2006).

64. Gorka, A. P. \& Schnermann, M. J. Harnessing cyanine photooxidation: from slowing photobleaching to near-IR uncaging. Curr. Opin. Chem. Biol. 33 , $117-125$ (2016)

65. Luciano, M. P. et al. A near-infrared light-mediated cleavable linker strategy using the heptamethine cyanine chromophore. Methods Enzymol. 641 245-275 (2020)

66. Gorka, A. P., Nani, R. R., Zhu, J., Mackem, S. \& Schnermann, M. J. A near-IR uncaging strategy based on cyanine photochemistry. J. Am. Chem. Soc. 136 , 14153-14159 (2014).

67. Nani, R. R. et al. In vivo activation of duocarmycinantibody conjugates by near-infrared light. ACS Cent. Sci. 3, 329-337 (2017)

68. Stackova, L. et al. Cyanine-flavonol hybrids for nearinfrared light-activated delivery of carbon monoxide. Chem. Eur. J. 26, 13184-13190 (2020).

69. Nani, R. R., Gorka, A. P., Nagaya, T., Kobayashi, H. \& Schnermann, M. J. Near-IR light-mediated cleavage of antibody-drug conjugates using cyanine photocages. Angew. Chem. Int. Ed. 54, 13635-13638 (2015).

70. Mitra, K. Platinum complexes as light promoted anticancer agents: a redefined strategy for controlled activation. Dalton Trans. 45, 19157-19171 (2016).
71. Zhang, Y. et al. Harnessing hypoxia-dependent cyanine photocages for in vivo precision drug release. Angew. Chem. Int. Ed. 60, 9553-9561 (2021).

72. Shell, T. A. \& Lawrence, D. S. Vitamin $B_{12}$ : a tunable long wavelength, light-responsive platform for launching therapeutic agents. Acc. Chem. Res. 48, 2866-2874 (2015)

73. Toda, M. J., Lodowski, P., Mamun, A. A., Jaworska, M $\&$ Kozlowski, P. M. Photolytic properties of the biologically active forms of vitamin $\mathrm{B}_{12}$. Coord. Chem. Rev. 385, 20-43 (2019).

74. Jones, A. R. The photochemistry and photobiology of vitamin $\mathrm{B}_{12}$. Photochem. Photobiol. Sci. 16, 820-834 (2017).

75. Martin, B. D. \& Finke, R. G. Methylcobalamin's fullvs. half-strength cobalt-carbon sigma bonds and bond dissociation enthalpies: $\mathrm{A}>10^{\wedge} 15 \mathrm{Co}-\mathrm{CH} 3$ homolysis rate enhancement following one-antibonding-electron reduction of methlycobalamin. J. Am. Chem. Soc. 114 585-592 (1992)

76. Halpern, J., Chan, M. S., Hanson, J., Roche, T. S. \& Topich, J. A. Detection and characterization of radical cations produced by one-electron chemical and electrochemical oxidations of organocobalt compounds J. Am. Chem. Soc. 97, 1606-1608 (1975).

77. Toscano, P. J. \& Marzilli, L. G. in Progress in Inorganic Chemistry (ed. Lippard, S. J.) 105-204 (Wiley, 1982).

78. Smith, W. J. et al. Cell-mediated assembly of phototherapeutics. Angew. Chem. Int. Ed. $\mathbf{5 3}$ 10945-10948 (2014).

79. Marvin, C. M. et al. On command drug delivery via cell-conveyed phototherapeutics. Small 15, e1901442 (2019).

80. Hughes, R. M. et al. Phototriggered secretion of membrane compartmentalized bioactive agents. Angew. Chem. Int. Ed. 55, 16080-16083 (2016).

81. Shell, T. A., Shell, J. R., Rodgers, Z. L. \& Lawrence, D. S. Tunable visible and near-IR photoactivation of lightresponsive compounds by using fluorophores as light-capturing antennas. Angew. Chem. Int. Ed. 53, 875-878 (2014)

82. O'Banion, C. P. et al. The plasma membrane as a reservoir, protective shield, and light-triggered launch pad for peptide therapeutics. Angew. Chem. Int. Ed. 55, 950-954 (2016)

83. Rehman, K. et al. Delivery of therapeutic proteins challenges and strategies. Curr. Drug Targets 17 , 1172-1188 (2016).

84. Lu, Y., Sun, W. \& Gu, Z. Stimuli-responsive nanomaterials for therapeutic protein delivery. J. Controlled Release 194, 1-19 (2014).

85. Vickerman, B. M., O'Banion, C. P., Tan, X. \& Lawrence, D. S. Light-controlled release of therapeutic proteins from red blood cells. ACS Cent. Sci. 7 , 93-103 (2021).

86. Henning, R. J. Therapeutic angiogenesis: angiogenic growth factors for ischemic heart disease. Future Cardiol. 12, 585-599 (2016).

87. Mari, C., Pierroz, V., Ferrari, S. \& Gasser, G. Combination of Ru(II) complexes and light: new frontiers in cancer therapy. Chem. Sci. 6, 2660-2686 (2015).

88. Li, A., Turro, C. \& Kodanko, J. J. Ru(II) polypyridyl complexes derived from tetradentate ancillary ligands for effective photocaging. Acc. Chem. Res. 51, 1415-1421 (2018).

89. Zayat, L., Calero, C., Albores, P., Baraldo, L. \& Etchenique, R. A new strategy for neurochemical photodelivery: metal-ligand heterolytic cleavage. J. Am. Chem. Soc. 125, 882-883 (2003)

90. Zayat, L., Filevich, O., Baraldo, L. M. \& Etchenique, R. Ruthenium polypyridyl phototriggers: from beginnings to perspectives. Phil. Trans. R. Soc. A 371, 20120330 (2013).

91. Zayat, L. et al. A new inorganic photolabile protecting group for highly efficient visible light GABA uncaging. Chembiochem 8, 2035-2038 (2007).

92. Havrylyuk, D., Stevens, K., Parkin, S. \& Glazer, E. C. Toward optimal Ru(II) photocages: balancing photochemistry, stability, and biocompatibility through fine tuning of steric, electronic, and physiochemical features. Inorg. Chem. 59, 1006-1013 (2020).

93. Li, A. et al. Illuminating cytochrome $\mathrm{P} 450$ binding: Ru(ii)-caged inhibitors of CYP17A1. Chem. Commun. 53, 3673-3676 (2017).

94. Lameijer, L. N. et al. A red-light-activated rutheniumcaged NAMPT inhibitor remains phototoxic in hypoxic cancer cells. Angew. Chem. Int. Ed. 56, 11549-11553 (2017).

95. Sharma, R. et al. Ruthenium tris(2-pyridylmethyl) amine as an effective photocaging group for nitriles. Inorg. Chem. 53, 3272-3274 (2014). 
96. van Rixel, V. H. S. et al. Photo-uncaging of a microtubule-targeted rigidin analogue in hypoxic cancer cells and in a xenograft mouse model. J. Am. Chem. Soc. 141, 18444-18454 (2019).

97. Loftus, L. M., Al-Afyouni, K. F. \& Turro, C. New Ru" scaffold for photoinduced ligand release with red light in the photodynamic therapy (PDT) window. Chem. Eur. J. 24, 11550-11553 (2018).

98. Loftus, L. M., Rack, J. J. \& Turro, C. Photoinduced ligand dissociation follows reverse energy gap law: nitrile photodissociation from low energy (3)MLCT excited states. Chem. Commun. 56, 4070-4073 (2020).

99. Lee, S. Y., Kim, C. Y. \& Nam, T. G. Ruthenium complexes as anticancer agents: a brief history and perspectives. Drug Des. Devel. Ther. 14, 5375-5392 (2020).

100. Anderson, E. D., Gorka, A. P. \& Schnermann, M. J. Near-infrared uncaging or photosensitizing dictated by oxygen tension. Nat. Commun. 7, 13378 (2016).

101. Chen, Y. \& Steinmetz, M. G. Photoactivation of amino-substituted 1,4-benzoquinones for release of carboxylate and phenolate leaving groups using visible light. J. Org. Chem. 71, 6053-6060 (2006)

102. Carling, C. J. et al. Efficient red light photo-uncaging of active molecules in water upon assembly into nanoparticles. Chem. Sci. 7, 2392-2398 (2016).

103. Chen, Y. \& Steinmetz, M. G. Photochemical cyclization with release of carboxylic acids and phenol from pyrrolidino-substituted 1,4-benzoquinones using visible light. Org. Lett. 7, 3729-3732 (2005).

104. Wang, X. \& Kalow, J. A. Rapid aqueous photouncaging by red light. Org. Lett. 20, 1716-1719 (2018).

105. Walton, D. P. \& Dougherty, D. A. A general strategy for visible-light decaging based on the quinone cis-alkenyl lock. Chem. Commun. 55, 4965-4968 (2019).

106. Jia, S., Fong, W.-K., Graham, B. \& Boyd, B. J. Photoswitchable molecules in long-wavelength lightresponsive drug delivery: from molecular design to applications. Chem. Mater. 30, 2873-2887 (2018).

107. Bleger, D. \& Hecht, S. Visible-light-activated molecular switches. Angew. Chem. Int. Ed. 54, 11338-11349 (2015)

108. Welleman, I. M., Hoorens, M. W. H., Feringa, B. L., Boersma, H. H. \& Szymański, W. Photoresponsive molecular tools for emerging applications of light in medicine. Chem. Sci. 11, 11672-11691 (2020).

109. Petermayer, C. \& Dube, H. Indigoid photoswitches: visible light responsive molecular tools. Acc. Chem. Res. 51, 1153-1163 (2018)

110. Dong, M., Babalhavaeji, A., Samanta, S., Beharry, A. A $\&$ Woolley, G. A. Red-shifting azobenzene photoswitches for in vivo use. Acc. Chem. Res. 48, 2662-2670 (2015)

111. Zhu, M. \& Zhou, H. Azobenzene-based small molecular photoswitches for protein modulation. Org. Biomol. Chem. 16, 8434-8445 (2018)

112. Hartley, G. S. The cis-form of azobenzene. Nature 140 281-281 (1937)

113. Konrad, D. B. et al. Computational design and synthesis of a deeply red-shifted and bistable azobenzene. J. Am Chem. Soc. 142, 6538-6547 (2020).

114. Martínez-López, D., Babalhavaeji, A., Sampedro, D. $\&$ Woolley, G. A. Synthesis and characterization of bis(4-amino-2-bromo-6-methoxy)azobenzene derivatives. Beilstein J. Org. Chem. 15, 3000-3008 (2019).

115. Konrad, D. B., Frank, J. A. \& Trauner, D. Synthesis of redshifted azobenzene photoswitches by late-stage functionalization. Chem. Eur. J. 22, 4364-4368 (2016).

116. Dong, M. et al. Near-infrared photoswitching of azobenzenes under physiological conditions. J. Am. Chem. Soc. 139, 13483-13486 (2017).

117. Siewertsen, R. et al. Highly efficient reversible $Z-E$ photoisomerization of a bridged azobenzene with visible light through resolved $\mathrm{S}_{1}\left(\mathrm{n} \pi^{*}\right)$ absorption bands. J. Am. Chem. Soc. 131, 15594-15595 (2009).

118. Trads, J. B. et al. Sign inversion in photopharmacology: incorporation of cyclic azobenzenes in photoswitchable potassium channel blockers and openers. Angew. Chem. Int. Ed. 58, 15421-15428 (2019).

119. Wegener, M., Hansen, M. J., Driessen, A. J. M., Szymanski, W. \& Feringa, B. L. Photocontrol of antibacterial activity: shifting from UV to red light activation. J. Am. Chem. Soc. 139, 17979-17986 (2017).

120. Wainwright, M. et al. Photoantimicrobials - are we afraid of the light? Lancet Infect. Dis. 17, 49-55 (2017).
121. Samanta, S. et al. Photoswitching azo compounds in vivo with red light. J. Am. Chem. Soc. 135 9777-9784 (2013).

122. Bleger, D., Schwarz, J., Brouwer, A. M. \& Hecht, S $o$-Fluoroazobenzenes as readily synthesized photoswitches offering nearly quantitative two-way isomerization with visible light. $J$. Am. Chem. Soc. 134, 20597-20600 (2012)

123. Wang, D., Wagner, M., Butt, H. J. \& Wu, S Supramolecular hydrogels constructed by red-lightresponsive host-guest interactions for photocontrolled protein release in deep tissue. Soft Matter 11, 7656-7662 (2015)

124. Wang, D. \& Wu, S. Red-light-responsive supramolecular valves for photocontrolled drug release from mesoporous nanoparticles. Langmuir 32 632-636 (2016)

125. Wen, J. et al. Diverse gatekeepers for mesoporous silica nanoparticle based drug delivery systems. Chem Soc. Rev. 46, 6024-6045 (2017).

126. Accardo, J. V., McClure, E. R., Mosquera, M. A ¿ Kalow, J. A. Using visible light to tune boronic acid-ester equilibria. J. Am. Chem. Soc. 142 19969-19979 (2020)

127. Accardo, J. V. \& Kalow, J. A. Reversibly tuning hydrogel stiffness through photocontrolled dynamic covalent crosslinks. Chem. Sci. 9, 5987-5993 (2018).

128. Hemmer, J. R. et al. Tunable visible and near infrared photoswitches. J. Am. Chem. Soc. 138, 13960-13966 (2016).

129. Poelma, S. O. et al. Controlled drug release to cancer cells from modular one-photon visible light-responsive micellar system. Chem. Commun. 52, 10525-10528 (2016).

130. Rifaie-Graham, O. et al. Wavelength-selective lightresponsive DASA-functionalized polymersome nanoreactors. J. Am. Chem. Soc. 140, 8027-8036 (2018).

131. Di Donato, M. et al. Shedding light on the photoisomerization pathway of donor-accepto stenhouse adducts. J. Am. Chem. Soc. 139 , 15596-15599 (2017).

132. Helmy, S. et al. Photoswitching using visible light: a new class of organic photochromic molecules. J. Am Chem. Soc. 136, 8169-8172 (2014).

133. Burke, P. W., Hoelzer, D., Park, J. H., Schmiegelow, K. $\&$ Douer, D. Managing toxicities with asparaginasebased therapies in adult ALL: summary of an ESMO Open-Cancer Horizons roundtable discussion. ESMO Open 5, 000858 (2020).

134. Olejniczak, J., Carling, C. J. \& Almutairi, A Photocontrolled release using one-photon absorption of visible or NIR light. J. Controlled Release 219, 18-30 (2015)

135. Zhang, Z. \& Tang, W. Drug metabolism in drug discovery and development. Acta Pharm. Sin. B 8 721-732 (2018)

136. Rizzo, F. \& Kehr, N. S. Recent advances in injectable hydrogels for controlled and local drug delivery. Adv. Healthc. Mater. 10, e2001341 (2020).

137. Scaranti, M., Cojocaru, E., Banerjee, S. \& Banerji, U. Exploiting the folate receptor a in oncology. Nat. Rev. Clin. Oncol. 17, 349-359 (2020).

138. Pettenuzzo, A., Pigot, R. \& Ronconi, L. Vitamin $\mathrm{B}_{12}$-metal conjugates for targeted chemotherapy and diagnosis: current status and future prospects. Eur. J. Inorg. Chem. 2017, 1625-1638 (2017).

139. Waibel, $R$ et al. New derivatives of vitamin $B 12$ show preferential targeting of tumours. Cancer Res. $\mathbf{6 8}$, 2904-2911 (2008)

140. Liu, S., Feng, X., Jin, R. \& Li, G. Tissue plasminogen activator-based nanothrombolysis for ischemic stroke. Expert Opin. Drug Deliv. 15, 173-184 (2018).

141. Gherasim, C., Lofgren, M. \& Banerjee, R. Navigating the $B_{12}$ road: assimilation, delivery, and disorders of cobalamin. J. Biol. Chem. 288, 13186-13193 (2013)

142. Jukes, Z., Morais, G. R., Loadman, P. M. \& Pors, K How can the potential of the duocarmycins be unlocked for cancer therapy? Drug Discov. Today 26, 577-584 (2020)

143. Kipshidze, N. et al. Viral coagulopathy in patients with COVID-19: treatment and care. Clin. Appl. Thromb. Hemost. 26, 1-7 (2020).

144. Muller, P. Y. \& Milton, M. N. The determination and interpretation of the therapeutic index in drug development. Nat. Rev. Drug Discov. 11, 751-761 (2012).

145. Heinemann, F., Karges, J. \& Gasser, G. Critical overview of the use of Ru(II) polypyridyl complexes as photosensitizers in one-photon and two-photon photodynamic therapy. Acc. Chem. Res. 50 2727-2736 (2017)

146. Weissleder, R. \& Ntziachristos, V. Shedding light onto live molecular targets. Nat. Med. 9, 123-128 (2003).

147. Kobayashi, H., Ogawa, M., Alford, R., Choyke, P. L. \& Urano, Y. New strategies for fluorescent probe design in medical diagnostic imaging. Chem. Rev. 110, 2620-2640 (2010).

148. Bianchini, G., Balko, J. M., Mayer, I. A., Sanders, M. E. \& Gianni, L. Triple-negative breast cancer: challenges and opportunities of a heterogeneous disease. Nat. Rev. Clin. Oncol. 13, 674-690 (2016).

149. Juiz, N. A., lovanna, J. \& Dusetti, N. Pancreatic cance heterogeneity can be explained beyond the genome. Front. Oncol. 9, 246 (2019).

150. Degl'Innocenti, A., di Leo, N. \& Ciofani, G. Genetic hallmarks and heterogeneity of glioblastoma in the single-cell omics era. Adv. Ther. 3, 1900152 (2020)

151. Tohme, S., Simmons, R. L. \& Tsung, A. Surgery for cancer: a trigger for metastases. Cancer Res. 77, 1548-1552 (2017).

152. Abuodeh, Y., Venkat, P. \& Kim, S. Systematic review of case reports on the abscopal effect. Curr. Probl. Cancer 40, 25-37 (2016).

153. Ngwa, W. et al. Using immunotherapy to boost the abscopal effect. Nat. Rev. Cancer 18, 313-322 (2018).

154. Nguyen, K. G. et al. Localized interleukin-12 for cancer immunotherapy. Front. Immunol. 11, 575597 (2020).

155. Reynders, K., Illidge, T., Siva, S., Chang, J. Y. $\S$ De Ruysscher, D. The abscopal effect of local radiotherapy: using immunotherapy to make a rare event clinically relevant. Cancer Treat. Rev. 41 503-510 (2015).

156. Baschant, U., Lane, N. E. \& Tuckermann, J. The multiple facets of glucocorticoid action in rheumatoid arthritis. Nat. Rev. Rheumatol. 8, 645-655 (2012).

157. Minter, R. R. et al. Protein engineering and preclinical development of a GM-CSF receptor antibody for the treatment of rheumatoid arthritis. Br. J. Pharmacol. 168, 200-211 (2013).

158. Wickenheisser, V. A. et al. Laser light therapy in inflammatory, musculoskeletal, and autoimmune disease. Curr. Allergy Asthma Rep. 19, 37 (2019).

159. Kadri, S. S. Key takeaways from the US CDC's 2019 antibiotic resistance threats report for frontline providers. Crit. Care Med. 48, 939-945 (2020).

160. Centers for Disease Control and Prevention. Antibiotic resistance threats in the United States. CDC https:// stacks.cdc.gov/view/cdc/82532 (2019).

161. Hamblin, M. R. \& Hasan, T. Photodynamic therapy: a new antimicrobial approach to infectious disease? Photochem. Photobiol. Sci. 3, 436-450 (2004).

162. World Health Organization. The top 10 causes of death (WHO, 2020).

163. Klegerman, M. E. Translational initiatives in thrombolytic therapy. Front. Med. 11, 1-19 (2017).

164. Zamanlu, M. et al. Recent advances in targeted delivery of tissue plasminogen activator for enhanced thrombolysis in ischaemic stroke. J. Drug Target. 26, 95-109 (2018).

\section{Acknowledgements}

We thank the following organizations for their generous financial support: the Rheumatology Research Foundation (T.K.T. and D.S.L.), the Eshelman Institute for Innovation (D.S.L.), the American Heart Association 18PRE33960038 (B.M.V) and the National Institutes of Health T32 CA71341 (E.M.Z.) and 1 R01 HL153744 (D.S.L.)

\section{Author contributions}

All authors researched data for the article, discussed the content, and reviewed and edited the manuscript before submission. B.M.V. and D.S.L. wrote the article.

\section{Competing interests}

The authors declare no competing interests.

\section{Peer review information}

Nature Reviews Chemistry thanks S. Bonnet, M. Schnermann and the other, anonymous, reviewers for their contribution to the peer review of this work.

\section{Publisher's note}

Springer Nature remains neutral with regard to jurisdictional claims in published maps and institutional affiliations.

C) Springer Nature Limited 2021, corrected publication 2021 